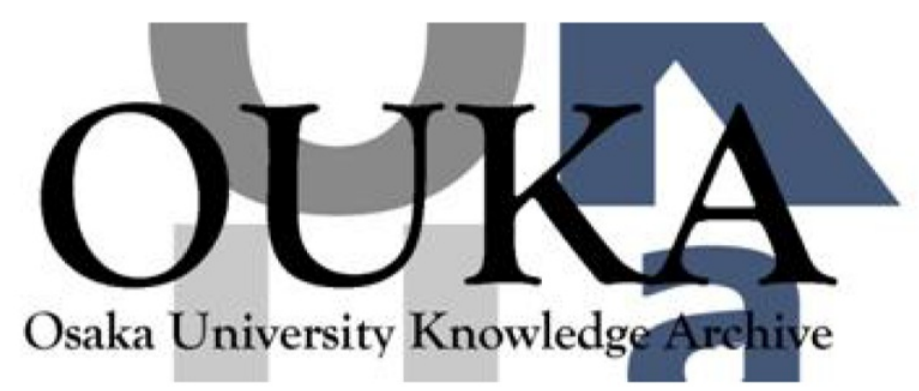

\begin{tabular}{|c|l|}
\hline Title & $\begin{array}{l}\text { QUASILINEAR DEGENERATE EVOLUTION EQUATIONS IN } \\
\text { BANACH SPACES }\end{array}$ \\
\hline Author(s) & Favini, Angelo; Yagi, Atsushi \\
\hline Citation & $\begin{array}{l}\text { Journal of Evolution Equations. 4(3) p. 421- } \\
\text { p. 449 }\end{array}$ \\
\hline Issue Date & $2004-09$ \\
\hline oaire:version VoR \\
\hline URL & https://hdl.handle.net/11094/24992 \\
\hline rights & \\
\hline Note & \\
\hline
\end{tabular}

Osaka University Knowledge Archive : OUKA

https://ir. Library. osaka-u. ac. jp/

Osaka University 


\title{
QUASILINEAR DEGENERATE EVOLUTION EQUATIONS IN BANACH SPACES
}

\author{
ANGELO FAVINI $(*)$ AND ATSUSHI YAGI $(* *)$
}

\begin{abstract}
The quasilinear degenerate evolution equation of parabolic type $\frac{d(M v)}{d t}+L(M v) v=F(M v), 0<t \leq T$ considered in a Banach space $X$ is written, putting $M v=u$, in the form $\frac{d u}{d t}+A(u) u \ni F(u), 0<t \leq T$, where $A(u)=L(u) M^{-1}$ are multivalued linear operators in $X$ for $u \in K, K$ being a bounded ball $\|u\|_{Z}<R$ in another Banach space $Z$ continuously embedded in $X$. Existence and uniqueness of the local solution for the related Cauchy problem are given. The results are applied to quasilinear elliptic-parabolic equations and systems.
\end{abstract}

\section{INTRODUCTION}

We are concerned with the Cauchy problem of a degenerate abstract evolution equation of parabolic type

$$
\left\{\begin{array}{l}
\frac{d M v}{d t}+L(M v) v=F(M v), \quad 0<t \leq T, \\
M v(0)=u_{0}
\end{array}\right.
$$

in a Banach space $X$. Here, $L(u)$ are closed linear operators in $X$ with some constant domain $\mathcal{D}(L(u)) \equiv \mathcal{D}_{L}$ for $u \in K=\left\{u \in Z ;\|u\|_{Z} \leq R\right\}, R>0$, where $Z$ is another Banach space such that $Z \subset X$ with continuous embedding. $M$ is a closed linear operator in $X$ with the domain $\mathcal{D}(M) \supset \mathcal{D}_{L}$ such that $M\left(\mathcal{D}_{L}\right) \subset Z$. $F(\cdot)$ is a nonlinear operator from $K$ into $X . u_{0} \in K$ is an initial value. $v=v(t)$ is an unknown function.

Cauchy problems of many concrete equations are formulated as those of abstract equations of the form (D.E), such as elliptic-parabolic equations, elliptic-parabolic systems, nonlinear equations of Sobolev type [14], semiconductor equations [15], and so on.

Like in our previous paper [3] (cf. also [11]) for linear problems, we rewrite the degenerate equation in (D.E) in the form $\frac{d u}{d t}+A(u) u \ni F(u)$ by changing unknown functions from $v=v(t)$ to $u=M v(t)$ and introducing multivalued linear operators $A(u)=L(u) M^{-1}, u \in K$, which act in $X$ with a constant domain $\mathcal{D}(A(u))=M\left(\mathcal{D}_{L}\right)$. In this way we have the Cauchy problem for a quasilinear

(*) Partially supported M. I. U. R. (Funds ex $60 \%$ ) and by University of Bologna Funds for selected research topics, the author is a menber of G. N. A. M. P. A. of INdAM.

(**) Partially supported by Grant-in-Aid for Scientific Research (No. 16340046) by Japan Society for the Promotion of Science. 
multivalued equation but of nondegenerate type

$$
\left\{\begin{array}{l}
\frac{d u}{d t}+A(u) u \ni F(u), \quad 0<t \leq T, \\
u(0)=u_{0}
\end{array}\right.
$$

Sobolevskii [8] (cf. also [12, Part 2] and [17]) has first studied such an abstract problem in the case where the coefficient operators $A(u)$ are all single valued and are the generators of analytic semigroups, that is $A(u)$ satisfy

$$
\left\|(\lambda-A(u))^{-1}\right\|_{\mathcal{L}(X)} \leq \frac{C}{(|\lambda|+1)^{1-\kappa}}, \quad \lambda \notin \Sigma, u \in K
$$

with the optimal exponent $\kappa=0$ and with some sectorial domain $\Sigma=\{\lambda \in$ $\mathbb{C} ;|\arg \lambda|<\phi\}, 0<\phi<\frac{\pi}{2}$. He in fact constructed, under suitable assumptions on $A(u), F(u)$ and $u_{0}$, a unique $X$-valued $\mathcal{C}^{1}$ local solution. We remark however that even if $L(u)$ are the generator of analytic semigroups, $A(u)=L(u) M^{-1}$ do not necessarily satisfy (1.1) with $\kappa=0$.

The first half of this paper is then devoted to studying the problem (E) with multivalued operators satisfying (1.1). We shall prove existence and uniqueness of $X$-valued $\mathrm{e}^{1}$ local solution by generalizing Sobolevskii's results on the basis of the previous works on multivalued linear evolution equations in [3].

In the second half we shall apply our abstract results to elliptic-parabolic equations and elliptic-parabolic systems. There is an enormous literature on the subject. We refer to the recent monograph by Showalter [16], see also [10]. In fact, most available results until now develop the basic approach by Brezis [2], where one sees the left hand side of (D.E) as the sum of two operators, the former being linear, the latter being (possibly nonlinear) monotone, and further assumptions allow to apply the theory by Bardos and Brezis [1]. On the other hand, such an approach forces to study the equations in some particular functional setting as $L^{p^{\prime}}(0, T ; W)$, where $W$ is either the dual space of a reflexive Banach space or a weighted space (depending on the operator $M$ ), $\frac{1}{p}+\frac{1}{p^{\prime}}=1, p \geq 2$.

The most recent main results on quasilinear degenerate evolution equations in [16, pp. 134-149] (see in particular Corollary 6.2 and Corollary 6.3) are concerned with the equation

$$
\frac{d}{d t}(B u)+A(t, u)=f(t), \quad \text { a. e. } t \in(0, T) .
$$

It is supposed that $B$ is a continuous, linear, symmetric and monotone operator from the reflexive separable Banach space $V$ to its dual $V^{\prime}$ and $A:[0, T] \times V \rightarrow V^{\prime}$ satisfies some appropriate continuity, monotonicity and coercivity assumptions ([16, p. 129]). The application of these results to quasilinear elliptic-parabolic equation is detailed in [16, Example 6.3]. For other results, we quote Kuttler [5] and $[6]$, too.

Here it will be shown that our approach allows to consider problems of this type having a nonlinearity (in $u$ ) in the right hand side of $(\mathrm{S})$, too, with a bit more restrictive assumptions on the data, due to the greater regularity in time of our solutions. 
The plan of the paper is as follows. In Section 2 we recall basic results on multivalued linear operators depending on time from [3] and [11], and prove new estimates for the evolution operators to be used in the subsequent sections. Section 3 is devoted to the problem (E). Some conditions given there are inspired by [8] and [9] too, and they guarantee the existence and uniqueness of the solutions to (E). In section 4 we apply such results to solve (D.E). Of course, if $L(u) \equiv L$ is independent of $u$, we can solve a semilinear degenerate differential equation. Sections 5 and 6 contain examples from partial differential equations of ellipticparabolic type to which our abstract theory applies. It is to be observed that unlike all previous literature, where the ambient space is a Sobolev space of negative exponent, here we can take $X=L^{2}(\Omega)$ (or, as shown in [4], $L^{p}(\Omega), 1<p<\infty$ ), when $\Omega$ is a bounded region in $\mathbb{R}^{n}$ with a smooth boundary.

Notation. Throughout the paper, $X$ denotes a complex Banach space whose norm is denoted by $\|\cdot\|_{X}$. If $Y$ is another Banach space, $\mathcal{L}(X, Y)$ is the space of all bounded linear operators from $X$ to $Y$ and $\|\cdot\|_{\mathcal{L}(X, Y)}$ denotes the uniform operator norm. $\mathcal{L}(X)$ is used for instead of $\mathcal{L}(X, X)$. An operator $A: X \rightarrow 2^{Y}$ having the two properties: $A u+A v \subset A(u+v), u, v \in X$ and $\lambda A u \subset A(\lambda u), \lambda \in \mathbb{C}, u \in X$ is called a multivalued linear operator from $\mathcal{D}(A)=\{u \in X ; A u \neq \emptyset\}$ to $Y$. For $u \in \mathcal{D}(A),\|A u\|_{X}=\inf \left\{\|f\|_{Y} ; f \in A u\right\}$. If $A: \mathcal{D}(A) \rightarrow Y$ is a multivalued linear operator, a single valued operator $A^{\circ}: \mathcal{D}(A) \rightarrow Y$ such that $A^{\circ} \subset A$ in the graph sense is called a section of $A$. With an arbitrary section $A^{\circ}$, it holds that $A u=A 0+A^{\circ} u, u \in \mathcal{D}(A)$.

If $I$ is a nonempty interval in $\mathbb{R}$ and $k$ is a nonnegative integer, $\mathcal{C}^{k}(I ; X)$ denotes the space of all $k$-times continuously differentiable functions with values in $X$ defined on $I$, where $\mathcal{C}^{0}(I ; X)=\mathcal{C}(I ; X)$. For $0<\mu<1, \mathcal{C}^{\mu}(I ; X)$ is the space of $\mu$-Hölder continuous functions with values in $X$ defined on $I . \mathcal{B}(I ; X)$ denotes the space of all bounded functions on $I$ with values in $X$.

\section{Multivalued linear equations}

We consider a family of multivalued linear operators $A(t), 0 \leq t \leq T$, acting in a Banach space $X$ which have a domain $\mathcal{D}(A(t)) \equiv \mathcal{D}$ independent of $t$. In the previous paper [3], we have already constructed the evolution operator $U(t, s)$ under the Assumptions (L.A.i,ii) and (L.Ex) below. The purpose of this section is then to review the basic properties of $U(t, s)$ and verify more refined ones which will be required in studying the multivalued quasilinear equation.

We make the following assumptions. For every $0 \leq t \leq T$, the spectral set $\sigma(A(t))$ of $A(t)$ is contained in a fixed open sectorial domain $\Sigma$,

$$
\sigma(A(t)) \subset \Sigma=\{\lambda \in \mathbb{C} ;|\arg \lambda|<\phi\},
$$

where $0<\phi<\frac{\pi}{2}$. And the resolvent satisfies the estimate

$$
\left\|(\lambda-A(t))^{-1}\right\|_{\mathcal{L}(X)} \leq \frac{M}{(|\lambda|+1)^{1-\kappa}}, \quad \lambda \notin \Sigma, 0 \leq t \leq T,
$$


with some exponent $0 \leq \kappa<1$ and a constant $M>0 . A(\cdot)$ satisfies a Hölder condition of the form

(L.A.ii) $\left\|A(t)\left\{A(t)^{-1}-A(s)^{-1}\right\} f\right\|_{X} \leq N|t-s|^{\mu}\|f\|_{X}, \quad f \in X, 0 \leq s, t \leq T$

with some exponent $0<\mu \leq 1$ and a constant $N>0$. The exponents satisfy the relation

$$
0 \leq 3 \kappa<\mu \leq 1
$$

Before introducing the evolution operator, let us first notice that (L.A.i) and (L.A.ii) imply the estimate

$$
\begin{aligned}
& \left\|A(t)^{\circ}(\lambda-A(t))^{-1}\left\{A(t)^{-1}-A(s)^{-1}\right\} f\right\|_{X} \\
& \quad \leq \frac{M N|t-s|^{\mu}}{(|\lambda|+1)^{1-\kappa}}\|f\|_{X}, \quad \lambda \notin \Sigma, f \in X .
\end{aligned}
$$

Here,

$$
A(t)^{\circ}(\lambda-A(t))^{-1}=\lambda(\lambda-A(t))^{-1}-1 \subset A(t)(\lambda-A(t))^{-1}
$$

denotes the linear section of $A(t)(\lambda-A(t))^{-1}$ introduced in [3, Theorem 2.7]. It is known that

$$
A(t)^{\circ}(\lambda-A(t))^{-1}=(\lambda-A(t))^{-1} A(t)^{\circ} \text { on } \mathcal{D},
$$

where $A(t)^{\circ}$ in the right hand side denotes an arbitrary section of $A(t)$ not necessarily linear. In fact, (2.1) is verified as follows. For $f \in X$,

$A(t)^{\circ}(\lambda-A(t))^{-1}\left\{A(t)^{-1}-A(s)^{-1}\right\} f=(\lambda-A(t))^{-1} A(t)^{\circ}\left\{A(t)^{-1}-A(s)^{-1}\right\} f$.

In addition, since

$$
(\lambda-A(t))^{-1} A(t)^{\circ}\left\{A(t)^{-1}-A(s)^{-1}\right\} f=(\lambda-A(t))^{-1} g
$$

with any $g \in A(t)\left\{A(t)^{-1}-A(s)^{-1}\right\} f$, it follows that

$$
\begin{aligned}
\left\|(\lambda-A(t))^{-1} A(t)^{\circ}\left\{A(t)^{-1}-A(s)^{-1}\right\} f\right\|_{X} \\
\quad \leq\left\|(\lambda-A(t))^{-1}\right\|_{\mathcal{L}(X)}\left\|A(t)\left\{A(t)^{-1}-A(s)^{-1}\right\} f\right\|_{X}, \quad f \in X .
\end{aligned}
$$

Therefore, (L.A.i) and (L.A.ii) imply (2.1).

In this theory we shall make an essential use of the Yosida approximation

$$
\begin{aligned}
A_{n}(t)=A(t)^{\circ} J_{n}(t) & =n\left\{1-J_{n}(t)\right\}, \quad n=1,2,3, \ldots, \\
J_{n}(t) & =\left(1+n^{-1} A(t)\right)^{-1}
\end{aligned}
$$

of $A(t) . A_{n}(t)$ are single valued bounded linear operators on $X$ with $\left\|A_{n}(t)\right\|_{\mathcal{L}(X)} \leq$ $C n^{1+\kappa}$. Since $A_{n}(t)^{-1}=A(t)^{-1}+n^{-1}$, we have

$$
A_{n}(t)\left\{A_{n}(t)^{-1}-A_{n}(s)^{-1}\right\} f=J_{n}(t) A(t)^{\circ}\left\{A(t)^{-1}-A(s)^{-1}\right\} f .
$$

Therefore, by $(2.2)$,

$$
\left\|A_{n}(t)\left\{A_{n}(t)^{-1}-A_{n}(s)^{-1}\right\} f\right\|_{X} \leq N\left\|J_{n}(t)\right\|_{\mathcal{L}(X)}|t-s|^{\mu}\|f\|_{X}, \quad f \in X .
$$

This shows that, as $\left\|J_{n}(t)\right\|_{\mathcal{L}(X)} \leq C n^{\kappa}$, the Hölder condition (L.A.ii) may not imply that of the Yosida approximation in any uniform sense. Such a difficulty is 
however overcome by the fact that (2.1) implies the same one for $A_{n}(t)$. In fact, since

$$
\begin{aligned}
& A_{n}(t)\left(\lambda-A_{n}(t)\right)^{-1}\left\{A_{n}(t)^{-1}-A_{n}(s)^{-1}\right\} f \\
& =\frac{n}{n-\lambda}\left(\frac{n}{n-\lambda}-A(t)\right)^{-1} A(t)^{\circ}\left\{A(t)^{-1}-A(s)^{-1}\right\} f,
\end{aligned}
$$

(2.1) yields that

$$
\left\|A_{n}(t)\left(\lambda-A_{n}(t)\right)^{-1}\left\{A_{n}(t)^{-1}-A_{n}(s)^{-1}\right\}\right\|_{\mathcal{L}(X)} \leq \frac{C|t-s|^{\mu}}{(|\lambda|+1)^{1-\kappa}}, \quad \lambda \notin \Sigma,
$$

see also $[3,(4.8)]$.

We shall also use the fractional powers of $A(t)$. For $\theta>\kappa$, the fractional power $A(t)^{-\theta}$ is defined by the Dunford integral

$$
A(t)^{-\theta}=\frac{1}{2 \pi i} \int_{\Gamma} \lambda^{-\theta}(\lambda-A(t))^{-1} d \lambda
$$

in $\mathcal{L}(X)$, where $\Gamma$ is an integral contour lying in $\mathbb{C}-\sigma(A(t))$. $A(t)^{\theta}, \theta>\kappa$, is then a multivalued linear operator in $X$. In particular, $A(t)^{1}=A(t)$. As $n \rightarrow \infty$, $A_{n}(t)^{-\theta}$ converges to $A(t)^{-\theta}$ in $\mathcal{L}(X)$.

(L.A.i) yields that for each $0 \leq t \leq T, A(t)$ generates an infinitely differential semigroup $e^{-\tau A(t)}, \tau \geq 0$, on $X$, see [3, Section 3]. For $\theta \geq 0$, a bounded linear operator on $X$ given by the integral

$$
\left\{A(t)^{\theta}\right\}^{\circ} e^{-\tau A(t)}=\frac{1}{2 \pi i} \int_{\Gamma} \lambda^{\theta} e^{-\tau \lambda}(\lambda-A(t))^{-1} d \lambda, \quad \tau>0,
$$

is introduced. Obviously, this operator is also obtained as a limit of $A_{n}(t)^{\theta} e^{-\tau A_{n}(t)}$ also. The following estimates

$$
\begin{gathered}
\left\|A_{n}(t)^{\theta} e^{-\tau A_{n}(t)} J_{n}(t)^{k}\right\|_{\mathcal{L}(X)} \leq C_{\theta} \tau^{-\theta-\kappa}, \quad \tau>0, k=0,1, \\
\left\|\left\{A(t)^{\theta}\right\}^{\circ} e^{-\tau A(t)}\right\|_{\mathcal{L}(X)} \leq C_{\theta} \tau^{-\theta-\kappa}, \quad \tau>0,
\end{gathered}
$$

are verified. If $\theta>\kappa,\left\{A(t)^{\theta}\right\}^{\circ} e^{-\tau A(t)}$ is really a linear section of the multivalued operator $A(t)^{\theta} e^{-\tau A(t)}$. For $\theta>\kappa$,

$$
\begin{array}{r}
\left\|\left\{e^{-\tau A(t)}-1\right\} A(t)^{-\theta}\right\|_{\mathcal{L}(X)}=\left\|\int_{0}^{\tau} A(t)^{\circ} e^{-\sigma A(t)} d \sigma A(t)^{-\theta}\right\|_{\mathcal{L}(X)} \\
=\left\|\int_{0}^{\tau}\left\{A(t)^{1-\theta}\right\}^{\circ} e^{-\sigma A(t)} d \sigma\right\|_{\mathcal{L}(X)} \leq C_{\theta} \tau^{\theta-\kappa}, \quad \tau>0 .
\end{array}
$$

According to [3, Theorem 4.1] (cf. also [11, Section 4.1]), under (L.A.i), (2.1), and (L.Ex), there exists an evolution operator $U(t, s), 0 \leq s \leq t \leq T$, for $A(t)$. $U(t, s)$ is in fact obtained as a limit of $U_{n}(t, s)$, where $U_{n}(t, s)$ is an evolution operator for $A_{n}(t)$. Moreover, $U_{n}(t, s) J_{n}(s)$ has the same limit as $U_{n}(t, s)$, that is, 
$U_{n}(t, s) J_{n}(s)$ also converges to $U(t, s)$. The estimates

$$
\begin{gathered}
\left\|U_{n}(t, s) J_{n}(s)^{k}\right\|_{\mathcal{L}(X)} \leq C(t-s)^{-\kappa}, \quad 0 \leq s<t \leq T, k=0,1, \\
\|U(t, s)\|_{\mathcal{L}(X)} \leq C(t-s)^{-\kappa}, \quad 0 \leq s<t \leq T
\end{gathered}
$$

hold. The convergence of $A_{n}(t) U_{n}(t, s)$ is also established, its limit being denoted by $A(t)^{\circ} U(t, s) . A(t)^{\circ} U(t, s)$ is a linear section of $A(t) U(t, s)$. The estimates

$$
\begin{array}{cc}
\left\|A_{n}(t) U_{n}(t, s)\right\|_{\mathcal{L}(X)} \leq C(t-s)^{-1-\kappa}, & 0 \leq s<t \leq T, \\
\left\|A(t)^{\circ} U(t, s)\right\|_{\mathcal{L}(X)} \leq C(t-s)^{-1-\kappa}, & 0 \leq s<t \leq T
\end{array}
$$

hold. Similarly, for $0<\theta<1$, a bounded linear operator $\left\{A(t)^{\theta}\right\}^{\circ} U(t, s)$ is defined as a limit of $A_{n}(t)^{\theta} U_{n}(t, s)$. The estimates

$$
\begin{gathered}
\left\|A_{n}(t)^{\theta} U_{n}(t, s)\right\|_{\mathcal{L}(X)} \leq C(t-s)^{-\theta-\kappa}, \quad 0 \leq s<t \leq T, 0 \leq \theta \leq 1, \\
\left\|\left\{A(t)^{\theta}\right\}^{\circ} U(t, s)\right\|_{\mathcal{L}(X)} \leq C(t-s)^{-\theta-\kappa}, \quad 0 \leq s<t \leq T, 0 \leq \theta \leq 1
\end{gathered}
$$

are verified by the moment inequality of the fractional powers. In addition, it is verified that

$$
\begin{aligned}
\left\|A_{n}(t)^{\theta} U_{n}(t, s) J_{n}(s)\right\|_{\mathcal{L}(X)} & \leq\left\|A_{n}(t)^{\theta} U_{n}\left(t, \frac{t+s}{2}\right)\right\|_{\mathcal{L}(X)} \\
\times\left\|U_{n}\left(\frac{t+s}{2}, s\right) J_{n}(s)\right\|_{\mathcal{L}(X)} & \leq C(t-s)^{-\theta-2 \kappa}, \quad 0 \leq s<t \leq T, 0<\theta \leq 1 .
\end{aligned}
$$

To obtain $(2.8)$ below, we notice from $[3,(4.10)]$ that

$$
\begin{aligned}
U_{n}(t, s) A_{n}(s)^{\theta}=A_{n}(s)^{\theta} e^{-(t-s) A_{n}(s)} & \\
& +\int_{s}^{t} U_{n}(t, \tau) A_{n}(\tau)\left\{A_{n}(\tau)^{-1}-A_{n}(s)^{-1}\right\} A_{n}(s)^{\theta+1} e^{-(\tau-s) A_{n}(s)} d \tau .
\end{aligned}
$$

By the same argument as for (2.2), we observe that

$$
\begin{aligned}
& \left\|U_{n}(t, \tau) A_{n}(\tau)\left\{A_{n}(\tau)^{-1}-A_{n}(s)^{-1}\right\} f\right\|_{X} \\
& \quad=\left\|U_{n}(t, \tau) J_{n}(\tau) A(\tau)^{\circ}\left\{A(\tau)^{-1}-A(s)^{-1}\right\} f\right\|_{X} \\
& \leq\left\|U_{n}(t, \tau) J_{n}(\tau)\right\|_{\mathcal{L}(X)}\left\|A(\tau)\left\{A(\tau)^{-1}-A(s)^{-1}\right\} f\right\|_{X}, \quad f \in X .
\end{aligned}
$$

Hence, for $0 \leq \theta<\mu-\kappa$,

$$
\begin{aligned}
& \left\|U_{n}(t, s) A_{n}(s)^{\theta}\right\|_{\mathcal{L}(X)} \leq C(t-s)^{-\theta-\kappa} \\
& \quad+C \int_{s}^{t}(t-\tau)^{-\kappa}(\tau-s)^{-\theta-1-\kappa+\mu} d \tau \leq C_{\theta}(t-s)^{-\theta-\kappa}, \quad 0 \leq s<t \leq T .
\end{aligned}
$$

We now prove some new estimates of $U_{n}(t, s)$ and $U(t, s)$.

Proposition 2.1. For $\kappa<\varphi \leq 1$,

$$
\begin{gathered}
\left\|A_{n}(t) U_{n}(t, s) A_{n}(s)^{-\varphi}\right\|_{\mathcal{L}(X)} \leq C_{\varphi}(t-s)^{\varphi-1-\kappa}, \quad 0 \leq s<t \leq T, \\
\left\|A(t)^{\circ} U(t, s) A(s)^{-\varphi}\right\|_{\mathcal{L}(X)} \leq C_{\varphi}(t-s)^{\varphi-1-\kappa}, \quad 0 \leq s<t \leq T .
\end{gathered}
$$


Proof. From

$$
\begin{aligned}
& U_{n}(t, s)=e^{-(t-s) A_{n}(t)} \\
& \quad-\int_{s}^{t} e^{-(t-\tau) A_{n}(t)} A_{n}(t)\left\{A_{n}(t)^{-1}-A_{n}(\tau)^{-1}\right\} A_{n}(\tau) U_{n}(\tau, s) d \tau,
\end{aligned}
$$

it follows that

$$
-\int_{s}^{t} A_{n}(t) e^{-(t-\tau) A_{n}(t)} J_{n}(t) A(t)^{\circ}\left\{A(t)^{-1}-A(\tau)^{-1}\right\} A_{n}(\tau) U_{n}(\tau, s) A_{n}(s)^{-\varphi} d \tau
$$

with any section $A(t)^{\circ} \subset A(t)$.

We here show the following lemma.

Lemma 2.1. For $0 \leq \theta \leq 1$ and $\kappa<\varphi \leq 1$,

$$
\begin{aligned}
\left\|\left\{A_{n}(t)^{\theta} e^{-\tau A_{n}(t)}-A_{n}(s)^{\theta} e^{-\tau A_{n}(s)}\right\} A_{n}(s)^{-\varphi}\right\|_{\mathcal{L}(X)} & \\
\leq & \begin{cases}C \Gamma(\theta-\varphi+2 \kappa) \tau^{\varphi-\theta-2 \kappa}|t-s|^{\mu}, & \text { if } \varphi<\theta+2 \kappa, \\
C\left\{\log \left(\tau^{-1}+1\right)+1\right\}|t-s|^{\mu}, & \text { if } \varphi=\theta+2 \kappa, \\
C(\varphi-\theta-2 \kappa)^{-1}|t-s|^{\mu}, & \text { if } \varphi>\theta+2 \kappa,\end{cases}
\end{aligned}
$$

where $\Gamma(\cdot)$ denotes the gamma function.

Letting $n \rightarrow \infty$, the same estimates are verified for the family $A(t)$, too.

Proof. We see that

$$
\begin{aligned}
& \left\{A_{n}(t)^{\theta} e^{-\tau A_{n}(t)}-A_{n}(s)^{\theta} e^{-\tau A_{n}(s)}\right\} A_{n}(s)^{-\varphi} \\
& =\frac{1}{2 \pi i} \int_{\Gamma} \lambda^{\theta} e^{-\tau \lambda}\left\{\left(\lambda-A_{n}(t)\right)^{-1}-\left(\lambda-A_{n}(s)\right)^{-1}\right\} A_{n}(s)^{-\varphi} d \lambda \\
& \left.=-\frac{1}{2 \pi i} \int_{\Gamma} \lambda^{\theta} e^{-\tau \lambda} A_{n}(t)\left(\lambda-A_{n}(t)\right)^{-1}\left\{A_{n}(t)^{-1}-A_{n}(s)\right)^{-1}\right\} \\
& \times A_{n}(s)^{1-\varphi}\left(\lambda-A_{n}(s)\right)^{-1} d \lambda,
\end{aligned}
$$

where $\Gamma$ is an integral contour: $\lambda=\rho e^{ \pm \phi i}, 0 \leq \rho<\infty$. Therefore, by (2.3), it follows that

$$
\begin{aligned}
\|\left\{A_{n}(t)^{\theta} e^{-\tau A_{n}(t)}-A_{n}(s)^{\theta} e^{-\tau A_{n}(s)}\right\} & A_{n}(s)^{-\varphi} \|_{\mathcal{L}(X)} \\
& \leq C \int_{\Gamma}(|\lambda|+1)^{\theta-\varphi+2 \kappa-1} e^{-\tau \Re \mathrm{e} \lambda}|d \lambda \| t-s|^{\mu} .
\end{aligned}
$$

If $\varphi<\theta+2 \kappa$, then

$$
\begin{aligned}
\int_{\Gamma}(|\lambda|+1)^{\theta-\varphi+2 \kappa-1} e^{-\tau \Re \mathrm{e} \lambda}|d \lambda| \\
\quad \leq C \int_{0}^{\infty} \rho^{\theta-\varphi+2 \kappa-1} e^{-\tau \rho \cos \phi} d \rho \leq C \tau^{\varphi-\theta-2 \kappa} \int_{0}^{\infty} \rho^{\theta-\varphi+2 \kappa-1} e^{-\rho} d \rho .
\end{aligned}
$$


If $\varphi=\theta+2 \kappa$, then

$$
\begin{aligned}
& \int_{\Gamma}(|\lambda|+1)^{\theta-\varphi+2 \kappa-1} e^{-\tau \Re \mathrm{e} \lambda}|d \lambda| \leq C \int_{0}^{\infty}(\rho+1)^{-1} e^{-\tau \rho \cos \phi} d \rho \\
& \leq C \int_{0}^{\tau^{-1}}(\rho+1)^{-1} d \rho+C \int_{\tau^{-1}}^{\infty} \rho^{-1} e^{-\tau \rho \cos \phi} d \rho .
\end{aligned}
$$

Hence the desired estimate is obtained. Similarly, if $\varphi>\theta+2 \kappa$, then

$$
\int_{\Gamma}(|\lambda|+1)^{\theta-\varphi+2 \kappa-1} e^{-\tau \Re \mathrm{e} \lambda}|d \lambda| \leq C \int_{0}^{\infty}(\rho+1)^{\theta-\varphi+2 \kappa-1} d \rho .
$$

Using this lemma with $\theta=1$, we have

$$
\begin{gathered}
\left\|A_{n}(t) e^{-(t-s) A_{n}(t)} A_{n}(s)^{-\varphi}\right\|_{\mathcal{L}(X)} \leq\left\|A_{n}(s)^{1-\varphi} e^{-(t-s) A_{n}(s)}\right\|_{\mathcal{L}(X)} \\
\quad+\left\|\left\{A_{n}(t) e^{-(t-s) A_{n}(t)}-A_{n}(s) e^{-(t-s) A_{n}(s)}\right\} A_{n}(s)^{-\varphi}\right\|_{\mathcal{L}(X)} \\
\leq C_{\varphi}\left[(t-s)^{\varphi-\kappa-1}+\log \left\{(t-s)^{-1}+1\right\}(t-s)^{\varphi-2 \kappa+\mu-1}\right] \leq C_{\varphi}(t-s)^{\varphi-\kappa-1} .
\end{gathered}
$$

Then, from (2.10), the following integral inequality

$$
\begin{aligned}
& \left\|A_{n}(t) U_{n}(t, s) A_{n}(s)^{-\varphi}\right\|_{\mathcal{L}(X)} \leq C_{\varphi}(t-s)^{\varphi-1-\kappa} \\
& \quad+C \int_{s}^{t}(t-\tau)^{\mu-1-\kappa}\left\|A_{n}(\tau) U_{n}(\tau, s) A_{n}(s)^{-\varphi}\right\|_{\mathcal{L}(X)} d \tau
\end{aligned}
$$

is obtained, which implies the first estimate (2.9).

Obviously the second estimate is an immediate consequence of the first one. Hence the proof of the proposition has been accomplished.

Proposition 2.2. For $0 \leq \theta<1-\kappa$ and $\theta+\kappa<\varphi \leq 1$,

$$
\begin{array}{ll}
\left\|A_{n}(t)^{\theta} U_{n}(t, s) A_{n}(s)^{-\varphi}\right\|_{\mathcal{L}(X)} \leq C_{\theta, \varphi}, & 0 \leq s<t \leq T, \\
\left\|\left\{A(t)^{\theta}\right\}^{\circ} U(t, s) A(s)^{-\varphi}\right\|_{\mathcal{L}(X)} \leq C_{\theta, \varphi}, & 0 \leq s<t \leq T .
\end{array}
$$

Proof. From (2.10) we can write that

$$
\begin{aligned}
& A_{n}(t)^{\theta} U_{n}(t, s) A_{n}(s)^{-\varphi}=A_{n}(t)^{\theta} e^{-(t-s) A_{n}(t)} A_{n}(s)^{-\varphi} \\
- & \int_{s}^{t} A_{n}(t)^{\theta} e^{-(t-\tau) A_{n}(t)} J_{n}(t) A(t)^{\circ}\left\{A(t)^{-1}-A(\tau)^{-1}\right\} A_{n}(\tau) U_{n}(\tau, s) A_{n}(s)^{-\varphi} d \tau .
\end{aligned}
$$

In addition, by (L.Ex), (2.5) and Lemma 2.1, we can observe that

$$
\begin{aligned}
& \left\|A_{n}(t)^{\theta} e^{-(t-s) A_{n}(t)} A_{n}(s)^{-\varphi}\right\|_{\mathcal{L}(X)} \\
& \leq\left\|\left\{A_{n}(t)^{\theta} e^{-(t-s) A_{n}(t)}-A_{n}(s)^{\theta} e^{-(t-s) A_{n}(s)}\right\} A_{n}(s)^{-\varphi}\right\|_{\mathcal{L}(X)} \\
& \quad+\left\|\left\{e^{-(t-s) A_{n}(s)}-1\right\} A_{n}(s)^{\theta-\varphi}\right\|_{\mathcal{L}(X)}+\left\|A_{n}(s)^{\theta-\varphi}\right\|_{\mathcal{L}(X)} \leq C_{\theta, \varphi} .
\end{aligned}
$$


Therefore, in view of (L.Ex), (2.4) and (2.9), we obtain that

$$
\begin{aligned}
& \| A_{n}(t)^{\theta} U_{n}(t, s) A_{n}(s)^{-\varphi} \|_{\mathcal{L}(X)} \\
& \leq C_{\theta, \varphi}\left\{1+\int_{s}^{t}(t-\tau)^{\mu-\theta-\kappa}(\tau-s)^{\varphi-\kappa-1} d \tau\right\} \\
& \quad \leq C_{\theta, \varphi}\left\{1+(t-s)^{\varphi-\theta-2 \kappa+\mu}\right\} \leq C_{\theta, \varphi} .
\end{aligned}
$$

The second estimate is an immediate consequence of this.

As to the difference of the evolution operator and the semigroup, we verify the following results.

Proposition 2.3. For $0 \leq \theta<1-2 \kappa$ and $\kappa<\varphi \leq 1$,

$$
\begin{aligned}
\left\|A_{n}(t)^{\theta}\left\{U_{n}(t, s)-e^{-(t-s) A_{n}(s)}\right\} A_{n}(s)^{-\varphi}\right\|_{\mathcal{L}(X)} & \\
& \leq C_{\theta, \varphi}(t-s)^{\varphi-\theta-3 \kappa+\mu}, \quad 0 \leq s<t \leq T .
\end{aligned}
$$

For $\kappa<\theta<1-2 \kappa$ and $\kappa<\varphi \leq 1$,

$$
\begin{aligned}
& \left\|A(t)^{\theta}\left\{U(t, s)-e^{-(t-s) A(s)}\right\} A(s)^{-\varphi} f\right\|_{X} \\
& \quad \leq C_{\theta, \varphi}(t-s)^{\varphi-\theta-3 \kappa+\mu}\|f\|_{X}, \quad 0 \leq s<t \leq T, \quad f \in X .
\end{aligned}
$$

Proof. Using [3, (4.10)] with $\rho=1$, we see that

$$
\begin{aligned}
& A_{n}(t)^{\theta}\left\{U_{n}(t, s)-e^{-(t-s) A_{n}(s)}\right\} A_{n}(s)^{-\varphi} \\
= & \int_{s}^{t} A_{n}(t)^{\theta} U_{n}(t, \tau) J_{n}(\tau) A(\tau)^{\circ}\left\{A(\tau)^{-1}-A(s)^{-1}\right\} A_{n}(s)^{1-\varphi} e^{-(t-s) A_{n}(s)} d \tau .
\end{aligned}
$$

Then, by (2.7) and (2.9), the norm of the right hand side is estimated by

$$
C_{\theta, \varphi} \int_{s}^{t}(t-\tau)^{-\theta-2 \kappa}(\tau-s)^{\varphi-1-\kappa+\mu} d \tau \leq C_{\theta, \varphi}(t-s)^{\varphi-\theta-3 \kappa+\mu} .
$$

Let $\kappa<\theta<1-2 \kappa$. Operating $A_{n}(t)^{-\theta}$ to (2.11) and letting $n \rightarrow \infty$ in the resulting equality, we obtain that

$$
\begin{aligned}
& \left\{U(t, s)-e^{-(t-s) A(s)}\right\} A(s)^{-\varphi} \\
& =A(t)^{-\theta} \int_{s}^{t}\left\{A(t)^{\theta}\right\}^{\circ} U(t, \tau) A(\tau)^{\circ}\left\{A(\tau)^{-1}-A(s)^{-1}\right\} A(s)^{1-\varphi} e^{-(t-s) A(s)} d \tau .
\end{aligned}
$$

From this the second estimate of the proposition is obtained.

We finally show a formula which gives a solution to the Cauchy problem of a multivalued linear equation

$$
\left\{\begin{array}{l}
\frac{d u}{d t}+A(t) u \ni F(t), \quad s<t \leq T \\
u(s)=u_{s}
\end{array}\right.
$$


in $X . s \in[0, T)$ is a fixed initial time. $F$ is a given Hölder continuous function on $[s, T]$ such that

$$
F \in \mathcal{C}^{\sigma}([s, T] ; X), \quad \sigma>\kappa .
$$

$u_{s}$ is an initial value in $X$ such that

$$
u_{s} \in \mathcal{D}\left(A(s)^{\gamma}\right), \quad \kappa<\gamma \leq 1
$$

As proved by [3, Theorem 4.1], there exists a unique solution to ( $\left.\mathrm{L} . \mathrm{E}_{s}\right)$ in the function space:

$$
u \in \mathcal{C}([s, T] ; X) \cap \mathcal{C}^{1}((s, T] ; X), \quad(t-s)^{1+\kappa-\gamma} \frac{d u}{d t} \in \mathcal{B}((s, T] ; X) .
$$

$u$ is in fact given by

$$
u(t)=U(t, s) u_{s}+\int_{s}^{t} U(t, \tau) F(\tau) d \tau, \quad s \leq t \leq T .
$$

Moreover, we can verify the following estimates

$$
\begin{gathered}
\left\|A_{n}(t) \int_{s}^{t} U_{n}(t, \tau) F(\tau) d \tau\right\|_{X} \leq C_{\sigma}(t-s)^{-\kappa}\|F\|_{\mathcal{C}^{\sigma}([s, T] ; X)}, \quad s<t \leq T, \\
\left\|A(t) \int_{s}^{t} U(t, \tau) F(\tau) d \tau\right\|_{X} \leq C_{\sigma}(t-s)^{-\kappa}\|F\|_{\mathcal{C}^{\sigma}([s, T] ; X)}, \quad s<t \leq T .
\end{gathered}
$$

Indeed,

$$
\begin{aligned}
& A_{n}(t) \int_{s}^{t} U_{n}(t, \tau) F(\tau) d \tau=\int_{s}^{t} A_{n}(t) U_{n}(t, \tau)\{F(\tau)-F(t)\} d \tau \\
+ & \int_{s}^{t}\left\{A_{n}(t) U_{n}(t, \tau)-A_{n}(t) e^{-(t-\tau) A_{n}(t)}\right\} F(t) d \tau+\left\{1-e^{-(t-s) A_{n}(t)}\right\} F(t) .
\end{aligned}
$$

Using the integral equation $[3,(4.12)]$, it is seen that

$$
\left\|A_{n}(t) U_{n}(t, s)-A_{n}(t) e^{-(t-s) A_{n}(t)}\right\|_{\mathcal{L}(X)} \leq C(t-s)^{-1-3 \kappa+\mu}, \quad 0 \leq s<t \leq T .
$$

Then (2.12) is obtained directly from (2.14). Operating $A_{n}(t)^{-1}$ to (2.14) and letting $n \rightarrow \infty$ in the resulting equation, we obtain that

$$
\begin{aligned}
& \int_{s}^{t} U(t, \tau) F(\tau) d \tau=A(t)^{-1}\left[\int_{s}^{t} A(t)^{\circ} U(t, \tau)\{F(\tau)-F(t)\} d \tau\right. \\
& \left.\quad+\int_{s}^{t}\left\{A(t)^{\circ} U(t, \tau)-A(t)^{\circ} e^{-(t-\tau) A(t)}\right\} F(t) d \tau+\left\{1-e^{-(t-s) A(t)}\right\} F(t)\right] .
\end{aligned}
$$

From this the estimate (2.13) is verified. 


\section{Quasilinear eVolution equations}

Let $X$ be a Banach space. We consider the Cauchy problem of a multivalued abstract evolution equation

$$
\left\{\begin{array}{l}
\frac{d u}{d t}+A(u) u \ni F(u), \quad 0<t \leq T, \\
u(0)=u_{0}
\end{array}\right.
$$

in $X$. Let $Z$ be a second Banach space continuously embedded in $X$ and let $K$ be an open ball of $Z$ such that

$$
K=\left\{u \in Z ;\|u\|_{Z}<R\right\}, \quad 0<R<\infty .
$$

For each $u \in K, A(u)$ is a multivalued linear operator of $X$ with domain $\mathcal{D}(A(u)) \equiv$ $\mathcal{D}$ which is constant in $u . F$ is a nonlinear operator from $K$ to $X . u_{0}$ is an initial value in $K$.

We make the following assumptions. The spectral set $\sigma(A(u))$ is contained in a fixed open sectorial region

$$
\sigma(A(u)) \subset \Sigma=\{\lambda \in \mathbb{C} ;|\arg \lambda|<\phi\},
$$

where $0<\phi<\frac{\pi}{2}$, and the resolvent satisfies

$$
\left\|(\lambda-A(u))^{-1}\right\|_{\mathcal{L}(X)} \leq \frac{M}{(|\lambda|+1)^{1-\kappa}}, \quad \lambda \notin \Sigma, u \in K
$$

with some exponent $0 \leq \kappa<1$ and a constant $M>0$ which are independent of u. $A(\cdot)$ satisfies a Lipschitz condition of the form

$$
\left\|A(u)\left\{A(u)^{-1}-A(v)^{-1}\right\} f\right\|_{X} \leq N\|u-v\|_{Z}\|f\|_{X}, \quad f \in X, \quad u, v \in K
$$

with a constant $N>0$. $F$ satisfies the Lipschitz condition

$$
\|F(u)-F(v)\|_{X} \leq L\|u-v\|_{Z}, \quad u, v \in K
$$

with a constant $L>0$. The spaces $X$ and $Z$ are as follows

$$
Z \subset X \text { with continuous embedding. }
$$

There is some exponent $\beta \in(\kappa, 1)$ such that, for every $u \in K, \mathcal{D}\left(A(u)^{\beta}\right) \subset Z$ with the estimate

$$
\|\widetilde{u}\|_{Z} \leq D\left\|A(u)^{\beta} \widetilde{u}\right\|_{X}, \quad \widetilde{u} \in \mathcal{D}\left(A(u)^{\beta}\right), u \in K,
$$

$D>0$ being some constant. $u_{0} \in K$ satisfies a compatibility condition of the form

$$
u_{0} \in \mathcal{D}\left(A\left(u_{0}\right)^{\gamma}\right)
$$

with some exponent $\gamma$ such that $\beta<\gamma \leq 1$. Finally, the exponents satisfy the relations

$$
0 \leq \kappa<\beta<\gamma \leq 1 \text { and } 5 \kappa+\beta<\gamma .
$$

As a matter of fact, (Ex) shows that $\kappa$ must be less than $\frac{1}{6}$.

Then, the following result is proved. 
Theorem 3.1. Under (A.i,ii), (F), (Sp.i,ii), (In), and (Ex), there exists a unique local solution to $(\mathrm{E})$ in the function space:

$$
\left\{\begin{array}{l}
u(t) \in \mathcal{D}\left(A(u(t)) \text { for } 0<t \leq T_{u_{0}} \text { and } u \in \mathcal{C}\left(\left[0, T_{u_{0}}\right] ; Z\right),\right. \\
u \in \mathcal{C}^{1}\left(\left(0, T_{u_{0}}\right] ; X\right) \text { and } t^{1+\kappa-\gamma} \frac{d u}{d t} \in \mathcal{B}\left(\left(0, T_{u_{0}}\right] ; X\right),
\end{array}\right.
$$

where $T_{u_{0}}>0$ is determined by the norms $\left\|u_{0}\right\|_{Z}$ and $\left\|A\left(u_{0}\right)^{\gamma} u_{0}\right\|_{X}$.

Proof. The proof consists of several steps. $C$ denotes a universal constant which is determined by the exponents and the initial constants. $f_{0}$ stands for an arbitrary element in $A\left(u_{0}\right)^{\gamma} u_{0}$.

Step 1. For $S$ such that $0<S \leq T$, we set a Banach space $z(S)=\mathcal{C}([0, S] ; Z)$ and a subset of $z(S)$ such that

$$
\begin{aligned}
\mathcal{K}(S)=\left\{u \in \mathcal{C}^{\mu}([0, S] ; Z) ; u(0)=u_{0},\right. \\
\left.\qquad \sup _{0 \leq s<t \leq S} \frac{\|u(t)-u(s)\|_{Z}}{|t-s|^{\mu}} \leq 1 \text { and } \sup _{0 \leq t \leq S}\|u(t)\|_{Z} \leq D_{u_{0}}\right\} .
\end{aligned}
$$

Here, $\mu$ is some fixed exponent so that $3 \kappa<\mu<\gamma-\beta-2 \kappa$, (Ex) shows that such a $\mu$ really exists. The constant $D_{u_{0}}$ is fixed so that

$$
\left\|u_{0}\right\|_{Z}<D_{u_{0}}<R \text {. }
$$

Clearly, $\mathcal{K}(S)$ is a nonempty closed subset of $\mathcal{Z}(S)$.

Step 2. For each $v \in \mathcal{K}(S)$, let us consider a linear problem

$$
\left\{\begin{array}{l}
\frac{d u}{d t}+A_{v}(t) u \ni F_{v}(t), \quad 0<t \leq S, \\
u(0)=u_{0}
\end{array}\right.
$$

where $A_{v}(t)=A(v(t))$ and $F_{v}(t)=F(v(t))$ for $0 \leq t \leq S$. It is easy to observe that $A_{v}(t)$ satisfies (L.A.i,ii) and (L.Ex) in Section 2 and that $F_{v} \in \mathcal{C}^{\mu}([0, S] ; X)$ and $u_{0}$ satisfy (L.F) and (L.In), respectively. Therefore, there exists a unique solution to (3.3) in the space

$$
u \in \mathcal{C}([0, S] ; X) \cap \mathcal{C}^{1}((0, S] ; X), t^{1+\kappa-\gamma} \frac{d u}{d t} \in \mathcal{B}((0, S] ; X),
$$

and the solution $u$ is given by

$$
u(t)=U_{v}(t, 0) u_{0}+\int_{0}^{t} U_{v}(t, s) F_{v}(s) d s, \quad 0 \leq t \leq S,
$$

where $U_{v}(t, s)$ denotes the evolution operator for the family of multivalued linear operators $A_{v}(t)=A(v(t))$.

We then arrive at defining a correspondence $\Phi$ from $\mathcal{K}(S)$ to $\mathcal{Z}(S)$ by setting $\Phi(v)(t)=u(t), 0 \leq t \leq S$, for each $v \in \mathcal{K}(S)$.

Step 3. If $S>0$ is sufficiently small, then $\Phi$ maps the set $\mathcal{K}(S)$ into itself. Indeed, for $u=\Phi(v)$, we write that

$$
u(t)=u_{0}+\left\{e^{-t A\left(u_{0}\right)}-1\right\} u_{0}+\left\{U_{v}(t, 0)-e^{-t A_{v}(0)}\right\} u_{0}+\int_{0}^{t} U_{v}(t, s) F_{v}(s) d s .
$$


Then, since $u_{0}=A\left(u_{0}\right)^{-\gamma} f_{0}$, it is seen by $(2,5)$ that

$$
\begin{aligned}
\left\|\left\{e^{-t A\left(u_{0}\right)}-1\right\} u_{0}\right\|_{Z} \leq & D\left\|A\left(u_{0}\right)^{\beta}\left\{e^{-t A\left(u_{0}\right)}-1\right\} A\left(u_{0}\right)^{-\gamma} f_{0}\right\|_{X} \\
& \leq C\left\|\left\{e^{-t A\left(u_{0}\right)}-1\right\} A\left(u_{0}\right)^{\beta-\gamma} f_{0}\right\|_{X} \leq C t^{\gamma-\beta-\kappa}\left\|f_{0}\right\|_{X} .
\end{aligned}
$$

Similarly, by Proposition 2.3,

$$
\begin{aligned}
\left\|\left\{U_{v}(t, 0)-e^{-t A_{v}(0)}\right\} u_{0}\right\|_{Z} \leq D \| A_{v}(t)^{\beta} & \left\{U_{v}(t, 0)-e^{-t A_{v}(0)}\right\} \\
& \times A_{v}(0)^{-\gamma} f_{0}\left\|_{X} \leq C t^{\gamma-\beta-3 \kappa+\mu}\right\| f_{0} \|_{X} .
\end{aligned}
$$

Finally, by (2.6),

$$
\left\|\int_{0}^{t} U_{v}(t, s) F_{v}(s) d s\right\|_{Z} \leq D\left\|\int_{0}^{t}\left\{A_{v}(t)^{\beta}\right\}^{\circ} U_{v}(t, s) F_{v}(s) d s\right\|_{X} \leq C t^{1-\beta-\kappa} .
$$

Therefore we obtain by definition (3.2) that

$$
\|u(t)\|_{Z} \leq\left\|u_{0}\right\|_{Z}+C\left(S^{\gamma-\beta-3 \kappa+\mu}+S^{1-\beta-\kappa}\right)\left(\left\|A\left(u_{0}\right)^{\gamma} u_{0}\right\|_{X}+1\right) \leq D_{u_{0}},
$$

provided that $S>0$ is sufficiently small. Note that $f_{0}$ denotes an arbitrary element of $A\left(u_{0}\right)^{\gamma} u_{0}$.

We next fix an exponent $\varphi$ so that

$$
\beta+3 \kappa<\beta+\kappa+\mu<\varphi<\gamma-\kappa \leq 1-\kappa,
$$

and notice that

$$
A_{v}(t)^{\varphi} u(t) \ni\left\{A_{v}(t)^{\varphi}\right\}^{\circ} U_{v}(t, 0) A_{v}(0)^{-\gamma} f_{0}+\int_{0}^{t}\left\{A_{v}(t)^{\varphi}\right\}^{\circ} U_{v}(t, s) F_{v}(s) d s=g_{v}(t) .
$$

By (2.6) and Proposition 2.2, $g_{v}(t)$ is shown to be uniformly bounded with

$$
\left\|g_{v}(t)\right\|_{X} \leq C_{\varphi}\left(\left\|A\left(u_{0}\right)^{\gamma} u_{0}\right\|_{X}+1\right), \quad 0 \leq t \leq S .
$$

Using $g_{v}(s)$, we can write that

$$
\begin{aligned}
u(t) & -u(s)=\left\{U_{v}(t, s)-1\right\} u(s)+\int_{s}^{t} U_{v}(t, \tau) F_{v}(\tau) d \tau \\
= & {\left[\left\{U_{v}(t, s)-e^{-(t-s) A_{v}(s)}\right\} A_{v}(s)^{-\varphi}\right.} \\
& \left.+\left\{e^{-(t-s) A_{v}(s)}-1\right\} A_{v}(s)^{-\varphi}\right] g_{v}(s) \\
& \quad+\int_{s}^{t} U_{v}(t, \tau) F_{v}(\tau) d \tau, \quad 0 \leq s<t \leq S .
\end{aligned}
$$

Then, by Proposition 2.3, it is seen that

$$
\left\|\left\{A_{v}(t)^{\beta}\right\}^{\circ}\left\{U_{v}(t, s)-e^{-(t-s) A_{v}(s)}\right\} A_{v}(s)^{-\varphi}\right\|_{\mathcal{L}(X)} \leq C_{\varphi}(t-s)^{\varphi-\beta-3 \kappa+\mu} .
$$

Similarly, by (2.5) and (2.6),

$$
\begin{gathered}
\left\|A_{v}(s)^{\beta}\left\{e^{-(t-s) A_{v}(s)}-1\right\} A_{v}(s)^{-\varphi}\right\|_{\mathcal{L}(X)} \leq C_{\varphi}(t-s)^{\varphi-\beta-\kappa}, \\
\left\|\int_{s}^{t}\left\{A_{v}(t)^{\beta}\right\}^{\circ} U_{v}(t, \tau) F_{v}(\tau) d \tau\right\|_{X} \leq C(t-s)^{1-\beta-\kappa} .
\end{gathered}
$$


Therefore, in view of (3.4), we observe that

$$
\begin{aligned}
\|u(t)-u(s)\|_{Z} \leq C_{\varphi}\left(S^{\varphi-\beta-3 \kappa}+\right. & S^{\varphi-\beta-\kappa-\mu} \\
& \left.\left.\quad+S^{1-\beta-\kappa-\mu}\right)\left(\| A\left(u_{0}\right)^{\gamma} u_{0}\right) \|_{X}+1\right)(t-s)^{\mu} .
\end{aligned}
$$

Therefore, in view of the definition of $\varphi$, we conclude that $\|u(t)-u(s)\|_{Z} \leq(t-s)^{\mu}$, provided $S>0$ is sufficiently small.

Step 4. If $S>0$ is sufficiently small, then the mapping $\Phi: \mathcal{K}(S) \rightarrow \mathcal{K}(S)$ is a contraction with respect to the norm $\|\cdot\|_{z(S)}$. Indeed, for $u_{i}=\Phi\left(v_{i}\right), v_{i} \in$ $\mathcal{K}(S), i=1,2$, we have

$$
\begin{aligned}
u_{1}(t) & -u_{2}(t)=\left\{U_{v_{1}}(t, 0)-U_{v_{2}}(t, 0)\right\} u_{0} \\
& +\int_{0}^{t}\left\{U_{v_{1}}(t, s)-U_{v_{2}}(t, s)\right\} F_{v_{2}}(s) d s+\int_{0}^{t} U_{v_{1}}(t, s)\left\{F_{v_{1}}(s)-F_{v_{2}}(s)\right\} d s .
\end{aligned}
$$

Here we establish the following lemma.

Lemma 3.1. We have

$$
\begin{aligned}
& \left\|A_{v_{1}}(t)^{\beta}\left\{U_{v_{1}}(t, 0)-U_{v_{2}}(t, 0)\right\} u_{0}\right\|_{X} \\
& \quad \leq C t^{\gamma-\beta-3 \kappa}\left\|A\left(u_{0}\right)^{\gamma} u_{0}\right\|_{X}\left\|v_{1}-v_{2}\right\|_{z(S)}, \quad 0 \leq t \leq S,
\end{aligned}
$$

and

$$
\begin{aligned}
& \| A_{v_{1}}(t)^{\beta} \int_{0}^{t}\left\{U_{v_{1}}(t, s)\right.\left.-U_{v_{2}}(t, s)\right\} F_{v_{2}}(s) d s \|_{X} \\
& \leq C t^{1-\beta-3 \kappa}\left\|F_{v_{2}}\right\|_{\mathcal{C}^{\mu}([0, t] ; X)}\left\|v_{1}-v_{2}\right\|_{z(S)}, \quad 0 \leq t \leq S .
\end{aligned}
$$

Proof. In order to verify these fundamental results, we have to employ the evolution operators $U_{v_{i}, n}(t, s)(i=1,2)$ for the families of the Yosida approximation $A_{v_{i}, n}(t)(i=1,2)$ of $A_{v_{i}}(t)$. Indeed we observe that

$$
\begin{aligned}
& A_{v_{1}, n}(t)^{\beta}\left\{U_{v_{1}, n}(t, 0)-U_{v_{2}, n}(t, 0)\right\} A_{v_{2}, n}(0)^{-\gamma}=\int_{0}^{t} A_{v_{1}, n}(t)^{\beta} U_{v_{1}, n}(t, s) \\
& \quad \times A_{v_{1}, n}(s)\left\{A_{v_{1}, n}(s)^{-1}-A_{v_{2}, n}(s)^{-1}\right\} A_{v_{2}, n}(s) U_{v_{2}, n}(s, 0) A_{v_{2}, n}(0)^{-\gamma} d s
\end{aligned}
$$

By the same argument as for (2.2), we can show by (2.7) that

$$
\begin{aligned}
& \left\|A_{v_{1}, n}(t)^{\beta} U_{v_{1}, n}(t, s) J_{v_{1}, n}(s) A_{v_{1}}(s)^{\circ}\left\{A_{v_{1}}(s)^{-1}-A_{v_{2}}(s)^{-1}\right\} f\right\|_{X} \\
& \leq C\left\|A_{v_{1}, n}(t)^{\beta} U_{v_{1}, n}(t, s) J_{v_{1}, n}(s)\right\|_{\mathcal{L}(X)} \| A_{v_{1}}(s)\left\{A_{v_{1}}(s)^{-1}-A_{v_{2}}(s)^{-1} f \|_{X}\right. \\
& \quad \leq C(t-s)^{-\beta-2 \kappa}\left\|v_{1}(s)-v_{2}(s)\right\|_{Z}\|f\|_{X}, \quad f \in X .
\end{aligned}
$$

where $A_{v_{1}}(s)^{\circ} \subset A_{v_{1}}(s)$ is an arbitrary section. Therefore,

$$
\begin{aligned}
\|\left\{A_{v_{1}}(t)^{\beta}\right\}^{\circ} U_{v_{1}}(t, s) & A_{v_{1}}(s)^{\circ}\left\{A_{v_{1}}(s)^{-1}-A_{v_{2}}(s)^{-1}\right\} f \|_{X} \\
& \leq C(t-s)^{-\beta-2 \kappa}\left\|v_{1}(s)-v_{2}(s)\right\|_{Z}\|f\|_{X}, \quad f \in X .
\end{aligned}
$$


Operating $A_{v_{1}, n}(t)^{-\beta}$ to $(3.5)$ and letting $n \rightarrow \infty$ in the resulting equality, we obtain that

$$
\begin{aligned}
\left\{U_{v_{1}}(t, 0)-U_{v_{2}}(t, 0)\right\} u_{0} & =A_{v_{1}}(t)^{-\beta} \int_{0}^{t}\left\{A_{v_{1}}(t)^{\beta}\right\}^{\circ} U_{v_{1}}(t, s) \\
\times & A_{v_{1}}(s)^{\circ}\left\{A_{v_{1}}(s)^{-1}-A_{v_{2}}(s)^{-1}\right\} A_{v_{2}}(s) U_{v_{2}}(s, 0) A_{v_{2}}(0)^{-\gamma} f_{0} d s .
\end{aligned}
$$

This yields that

$$
\begin{aligned}
& \left\|A_{v_{1}}(t)^{\beta}\left\{U_{v_{1}}(t, 0)-U_{v_{2}}(t, 0)\right\} u_{0}\right\|_{X} \\
& \quad \leq C \int_{0}^{t}(t-s)^{-\beta-2 \kappa} s^{\gamma-1-\kappa} d s\left\|v_{1}-v_{2}\right\|_{z(S)}\left\|f_{0}\right\|_{X} .
\end{aligned}
$$

Since $f_{0} \in A\left(u_{0}\right)^{\gamma} u_{0}$ is arbitrary, we obtain the first estimation.

Next, we can write that

$$
\begin{gathered}
A_{v_{1}, n}(t)^{\beta} \int_{0}^{t}\left\{U_{v_{1}, n}(t, s)-U_{v_{2}, n}(t, s)\right\} F_{v_{2}}(s) d s=\int_{0}^{t} \int_{s}^{t} A_{v_{1}, n}(t)^{\beta} U_{v_{1}, n}(t, \tau) \\
\times A_{v_{1}, n}(\tau)\left\{A_{v_{1}, n}(\tau)^{-1}-A_{v_{2}, n}(\tau)^{-1}\right\} A_{v_{2}, n}(\tau) U_{v_{2}, n}(\tau, s) F_{v_{2}}(s) d \tau d s \\
=\int_{0}^{t} A_{v_{1}, n}(t)^{\beta} U_{v_{1}, n}(t, \tau) A_{v_{1}, n}(\tau)\left\{A_{v_{1}, n}(\tau)^{-1}-A_{v_{2}, n}(\tau)^{-1}\right\} \\
\times A_{v_{2}, n}(\tau) \int_{0}^{\tau} U_{v_{2}, n}(\tau, s) F_{v_{2}}(s) d s d \tau .
\end{gathered}
$$

From $(2.12), A_{v_{2}, n}(\tau) \int_{0}^{\tau} U_{v_{2}, n}(\tau, s) F_{v_{2}}(s) d s$ satisfies the uniform estimate

$$
\left\|A_{v_{2}, n}(\tau) \int_{0}^{\tau} U_{v_{2}, n}(\tau, s) F_{v_{2}}(s) d s\right\|_{X} \leq C \tau^{-\kappa}\left\|F_{v_{2}}\right\|_{\mathcal{C}^{\mu}([0, S] ; X)},
$$

and converges as $n \rightarrow \infty$ to a continuous function $g(\tau)$ on $(0, S]$. Then, we obtain in the same way as above that

$$
\begin{aligned}
& \int_{0}^{t}\left\{U_{v_{1}}(t, s)-U_{v_{2}}(t, s)\right\} F_{v_{2}}(s) d s \\
& \quad=A_{v_{1}}(t)^{-\beta} \int_{0}^{t}\left\{A_{v_{1}}(t)^{\beta}\right\}^{\circ} U_{v_{1}}(t, \tau) A_{v_{1}}(\tau)^{\circ}\left\{A_{v_{1}}(\tau)^{-1}-A_{v_{2}}(\tau)^{-1}\right\} g(\tau) d \tau .
\end{aligned}
$$

Therefore,

$$
\begin{aligned}
& \| A_{v_{1}}(t)^{\beta} \int_{0}^{t}\left\{U_{v_{1}}(t, s)\right.\left.-U_{v_{2}}(t, s)\right\} F_{v_{2}}(s) d s \|_{X} \\
& \leq C \int_{0}^{t}(t-\tau)^{-\beta-2 \kappa} \tau^{-\kappa} d \tau\left\|F_{v_{2}}\right\|_{\mathcal{C}^{\mu}([0, S] ; X)}\left\|v_{1}-v_{2}\right\|_{\mathcal{Z}(S)} .
\end{aligned}
$$

Hence we verify the second estimate of the lemma.

Let us now complete the proof of this Step. It is easy to see that

$$
\left\|A_{v_{1}}(t)^{\beta} \int_{0}^{t} U_{v_{1}}(t, s)\left\{F_{v_{1}}(s)-F_{v_{2}}(s)\right\} d s\right\|_{X} \leq C t^{1-\beta-\kappa}\left\|v_{1}-v_{2}\right\|_{z(S)} .
$$


This together with the lemma then yields that

$$
\left\|u_{1}(t)-u_{2}(t)\right\|_{Z} \leq C S^{\gamma-\beta-3 \kappa}\left(\left\|A\left(u_{0}\right)^{\gamma} u_{0}\right\|_{X}+1\right)\left\|v_{1}-v_{2}\right\|_{z(S)}, \quad 0 \leq t \leq S .
$$

Hence, we have verified that $\Phi$ is a contraction, provided $S>0$ is sufficiently small.

Step 5. Take a $T_{u_{0}}=S>0$ in such a way that the results of Steps 3 and 4 are valid. Then, there exist a unique fixed point $u \in \mathcal{K}(S)$ of $\Phi$. Since $u$ satisfies the formula

$$
u(t)=U_{u}(t, 0) u_{0}+\int_{0}^{t} U_{u}(t, s) F_{u}(s) d s, \quad 0 \leq t \leq S,
$$

$u$ is shown to be a solution to (E) which belongs to the space (3.1).

Step 6. Finally we verify the uniqueness of solution. Let $u$ be the solution constructed above. We consider the Yosida approximation $A_{u, n}(t)$ of the operator $A_{u}(t)$ and the evolution operator $U_{u, n}(t, s)$ corresponding to $A_{u, n}(t)$. Let $\widetilde{u}$ be any other solution to (E) in the space (3.1). Then, for $0<t<S\left(\leq T_{u_{0}}\right)$,

$$
\frac{\partial}{\partial s} U_{u, n}(t, s) \widetilde{u}(s)=U_{u, n}(t, s)\left\{A_{u, n}(s) \widetilde{u}(s)-\widetilde{g}(s)\right\}+U_{u, n}(t, s) F_{\widetilde{u}}(s), \quad 0<s<t,
$$

where $\widetilde{g}(s) \in A_{\widetilde{u}}(s) \widetilde{u}(s)$ with $\frac{d \widetilde{u}}{d s}+\widetilde{g}(s)=F_{\widetilde{u}}(s)$. Integrating this identity in $s \in(0, t)$ and operating $A_{u, n}(t)^{\beta}$ to the resulting one yield that

$$
\begin{array}{r}
A_{u, n}(t)^{\beta}\left\{\widetilde{u}(t)-u_{n}(t)\right\}=\int_{0}^{t} A_{u, n}(t)^{\beta} U_{u, n}(t, s) \\
\quad \times A_{u, n}(s)\left\{A_{\widetilde{u}}(s)^{-1}-A_{u, n}(s)^{-1}\right\} \widetilde{g}(s) d s \\
\quad+\int_{0}^{t} A_{u, n}(t)^{\beta} U_{u, n}(t, s)\left\{F_{\widetilde{u}}(s)-F_{u}(s)\right\} d s,
\end{array}
$$

where $u_{n}(t)=U_{u, n}(t, 0) u_{0}+\int_{0}^{t} U_{u, n}(t, s) F_{u}(s) d s$.

We are concerned with the limit as $n \rightarrow \infty$. By the same method as in Step 4, it is in fact verified that

$$
\begin{array}{r}
\widetilde{u}(t)-u(t)=A_{u}(t)^{-\beta}\left[\int_{0}^{t}\left\{A_{u}(t)^{\beta}\right\}^{\circ} U_{u}(t, s) A_{u}(s)^{\circ}\left\{A_{\widetilde{u}}(s)^{-1}-A_{u}(s)^{-1}\right\}\right. \\
\left.\times \widetilde{g}(s) d s+\int_{0}^{t}\left\{A_{u}(t)^{\beta}\right\}^{\circ} U_{u}(t, s)\left\{F_{\widetilde{u}}(s)-F_{u}(s)\right\} d s\right] \\
-\lim _{n \rightarrow \infty} n^{-1} \int_{0}^{t} U_{u, n}(t, s) A_{u, n}(s) \widetilde{g}(s) d s .
\end{array}
$$

Moreover, we observe the following fact.

\section{Lemma 3.2.}

$$
\lim _{n \rightarrow \infty} n^{-1} \int_{0}^{t} U_{u, n}(t, s) A_{u, n}(s) \widetilde{g}(s) d s=0
$$


Proof. For any $\rho \in(0, \mu-\kappa)$, we have by (2.8) that

$$
\begin{aligned}
\left\|U_{u, n}(t, s) A_{u, n}(s)\right\|_{\mathcal{L}(X)} \leq\left\|U_{u, n}(t, s) A_{u, n}(s)^{\rho}\right\|_{\mathcal{L}(X)}\left\|A_{u, n}(s)^{1-\rho}\right\|_{\mathcal{L}(X)} \\
\quad \leq C_{\rho}(t-s)^{-\rho-\kappa}\left\|A_{u, n}(s)\right\|_{\mathcal{L}(X)}^{1-\rho} \leq C_{\rho} n^{(1+\kappa)(1-\rho)}(t-s)^{-\rho-\kappa} .
\end{aligned}
$$

In addition, from (3.1), $\widetilde{g}$ satisfies that $\|\widetilde{g}(s)\|_{X} \leq C_{\widetilde{u}} s^{\gamma-\kappa-1}$. Therefore,

$$
\begin{aligned}
\| \int_{0}^{t} U_{u, n}(t, s) A_{u, n}(s) & \widetilde{g}(s) d s \|_{X} \leq C_{\rho} C_{\widetilde{u}} n^{(1+\kappa)(1-\rho)} \\
& \times \int_{0}^{t}(t-s)^{-\rho-\kappa} s^{\gamma-\kappa-1} d s \leq C_{\rho} C_{\widetilde{u}} n^{(1+\kappa)(1-\rho)} t^{\gamma-\rho-2 \kappa} .
\end{aligned}
$$

It then suffices to take a $\rho$ so that $\frac{\kappa}{1+\kappa}<\rho<\mu-\kappa$. Since $\frac{\kappa}{1+\kappa}+\kappa \leq 3 \kappa(<\mu)$ for $0 \leq \kappa<\frac{1}{6}$, it is clearly possible to take such a $\rho$.

In view of (3.6), we verify from (3.7) that

$$
\left\|A_{u}(t)^{\beta}\{\widetilde{u}(t)-u(t)\}\right\|_{X} \leq C_{\widetilde{u}} S^{\gamma-\beta-3 \kappa}\|\widetilde{u}-u\|_{\mathcal{Z}(S)}, \quad 0 \leq t \leq S .
$$

This in turn shows that $\widetilde{u}(t)=u(t)$ for all $t \in[0, S]$ if $S>0$ is sufficiently small. As a matter of fact, we have shown by this argument that the set $\left\{S \in\left(0, T_{U_{0}}\right] ; \widetilde{u}(t)=\right.$ $u(t)$ for all $t \in[0, S]\}$ is nonempty and open in $\left(0, T_{U_{0}}\right]$. On the other hand, it is clear that the set is closed. Therefore, $\widetilde{u}(t)=u(t)$ for all $t \in\left[0, T_{U_{0}}\right]$.

Remark 3.1. As shown in the proof, $T_{u_{0}}$ is determined by the norm

$$
\left\|A\left(u_{0}\right)^{\gamma} u_{0}\right\|_{X}=\inf \left\{\left\|f_{0}\right\|_{X} ; f_{0} \in A\left(u_{0}\right)^{\gamma} u_{0}\right\} .
$$

This then means that the global existence of solution to (E) will be established if we can verify a priori estimates $\|u(t)\|_{Z}<R$ and $\left\|A(u(t))^{\gamma} u(t)\right\|_{X} \leq C$ for every local solution.

\section{Degenerate Abstract Evolution Equations}

We consider the Cauchy problem of a degenerate abstract evolution equation

$$
\left\{\begin{array}{l}
\frac{d M v}{d t}+L(M v) v=F(M v), \quad 0<t \leq T, \\
M v(0)=u_{0}
\end{array}\right.
$$

in a Banach space $X$. Let $Z \subset X$ be the second Banach space continuously embedded in $X$ and $K$ be a bounded subset of $Z$ such that

$$
K=\left\{u \in Z ;\|u\|_{Z}<R\right\}, 0<R<\infty .
$$

For each $u \in K, L(u)$ is a densely defined closed linear operator of $X$ with constant domain $\mathcal{D}(L(u)) \equiv \mathcal{D}_{L} . M$ is a closed linear operator of $X$ with domain $\mathcal{D}(M) \supset$ $\mathcal{D}_{L}$, and $M$ maps $\mathcal{D}_{L}$ into $Z$. $F$ is a nonlinear operator from $K$ into $X . u_{0} \in K$ is an initial value of the problem. $v=v(t)$ is the unknown function. 
We make the following assumptions. For every $u \in K$, the $M$-modified spectral set $\sigma_{M}(L(u))$ is contained in a fixed open sectorial region

$$
\sigma_{M}(L(u)) \subset \Sigma=\{\lambda \in \mathbb{C} ;|\arg \lambda|<\phi\},
$$

where $0<\phi<\frac{\pi}{2}$, and the $M$-modified resolvent satisfies

$$
\left\|M(\lambda M-L(u))^{-1}\right\|_{\mathcal{L}(X)} \leq \frac{C}{(|\lambda|+1)^{1-\kappa}}, \quad \lambda \notin \Sigma, u \in K
$$

with some exponent $0 \leq \kappa<1$ and a constant $C>0$ which are independent of $u$. The $M$-modified resolvent also satisfies

$$
\left\|M(\lambda M-L(u))^{-1}\right\|_{\mathcal{L}(X, Z)} \leq \frac{C}{(|\lambda|+1)^{1-\rho}}, \quad \lambda \leq 0, u \in K
$$

with some exponent $\kappa \leq \rho<1$ and a constant $C>0$ independent of $u$. $L(u)$ satisfies the Lipschitz condition

$$
\left\|L(u)\left\{L(u)^{-1}-L(\widetilde{u})^{-1}\right\}\right\|_{\mathcal{L}(X)} \leq C\|u-\widetilde{u}\|_{Z}, \quad u, \widetilde{u} \in K
$$

with some constant $C>0$. F also satisfies the Lipschitz condition

$$
\|F(u)-F(\widetilde{u})\|_{X} \leq C\|u-\widetilde{u}\|_{Z}, \quad u, \widetilde{u} \in K
$$

with some constant $C>0$.

We set $u(t)=M v(t)$ and rewrite (D.E) in the form

$$
\left\{\begin{array}{l}
\frac{d u}{d t}+L(u) M^{-1} u \ni F(u), \quad 0<t \leq T, \\
u(0)=u_{0} .
\end{array}\right.
$$

Here, $L(u) M^{-1}=A(u)$ is a multivalued linear operator defined for $u \in K$ with the constant domain $\mathcal{D}(A(u))=M\left(\mathcal{D}_{L}\right)$. Our goal is then to apply the Theorem 3.1 to the present Cauchy problem.

According to [3, Theorem1.14], if $\lambda \notin \sigma_{M}(L(u))$, then $\lambda \notin \sigma(A(u))$, and it holds that

Therefore, (D.A.i) yields that

$$
M(\lambda M-L(u))^{-1}=(\lambda-A(u))^{-1}, \quad \lambda \notin \sigma_{M}(L(u)) .
$$

$$
\left\|(\lambda-A(u))^{-1}\right\|_{\mathcal{L}(X)} \leq \frac{C}{(|\lambda|+1)^{1-\kappa}}, \quad \lambda \notin \Sigma .
$$

For $u, \widetilde{u} \in K$,

$$
\left\{A(u)^{-1}-A(\widetilde{u})^{-1}\right\} f=M\left\{L(u)^{-1}-L(\widetilde{u})^{-1}\right\} f \in \mathcal{D}(A(u)), \quad f \in X .
$$

In addition,

$$
L(u)\left\{L(u)^{-1}-L(\widetilde{u})^{-1}\right\} f \in A(u)\left\{A(u)^{-1}-A(\widetilde{u})^{-1}\right\} f .
$$

Therefore, it follows from (D.A.iii) that

$$
\begin{aligned}
\| A(u)\left\{A(u)^{-1}\right. & \left.-A(\widetilde{u})^{-1}\right\} f \|_{X} \\
& \leq\left\|L(u)\left\{L(u)^{-1}-L(\widetilde{u})^{-1}\right\} f\right\|_{X} \leq C\|u-\widetilde{u}\|_{Z}\|f\|_{X}, \quad f \in X
\end{aligned}
$$

Hence, (A.i,ii) in the preceding section have been verified. 
For $\kappa<\beta<1$ it is known that

$$
A(u)^{-\beta} f=\frac{\sin \pi \beta}{\pi} \int_{0}^{\infty} \lambda^{-\beta}(\lambda+A(u))^{-1} f d \lambda, \quad f \in X .
$$

From (D.A.ii) it is seen that

$$
\left\|(\lambda+A(u))^{-1}\right\|_{\mathcal{L}(X, Z)} \leq \frac{C}{(\lambda+1)^{1-\rho}}, \quad \lambda \geq 0 .
$$

Therefore, for any $\beta$ such that $\rho<\beta<1$,

$$
\left\|A(u)^{-\beta} f\right\|_{Z} \leq \frac{1}{\pi} \int_{0}^{\infty} \lambda^{-\beta}(\lambda+1)^{\rho-1} d \lambda\|f\|_{X} \leq C\|f\|_{X} .
$$

Setting $\widetilde{u}=A(u)^{-\beta} f$, we observe that

$$
\|\widetilde{u}\|_{Z} \leq C\|f\|_{X}
$$

For a given $\widetilde{u} \in \mathcal{D}\left(A(u)^{\beta}\right)$, this is true for any $f \in A(u)^{\beta} \widetilde{u}$. Hence, (Sp.ii) is fulfilled with any $\beta \in(\rho, 1)$.

For (In), we assume that $u_{0} \in K$ and $u_{0}$ satisfies a compatibility condition of the form

$$
u_{0} \in \mathcal{D}\left(\left\{L\left(u_{0}\right) M^{-1}\right\}^{\gamma}\right)
$$

with some exponent $0<\gamma \leq 1$. For the exponents we assume the relations

$$
0 \leq \kappa \leq \rho<\gamma \leq 1 \text { and } 5 \kappa+\rho<\gamma .
$$

It is then possible to take the exponent $\beta$ in such a way that (Sp.ii) and (Ex) hold.

We have thus found out the conditions to be assumed to apply Theorem 3.1 and obtained the main result of the paper.

Theorem 4.1. Under (D.A.i,ii,iii), (D.F), (D.Ex), and (D.In), there exists a unique local solution to (D.E) in the function space

$$
\left\{\begin{array}{l}
M v \in \mathcal{C}\left(\left[0, T_{u_{0}}\right] ; Z\right) \cap \mathcal{C}^{1}\left(\left(0, T_{u_{0}}\right] ; X\right), \\
v \in \mathcal{C}\left(\left(0, T_{u_{0}}\right] ; \mathcal{D}_{L}\right), t^{1+\kappa-\gamma} L(M v) v \in \mathcal{B}\left(\left(0, T_{u_{0}}\right] ; X\right),
\end{array}\right.
$$

where $T_{u_{0}}>0$ is determined by the norms $\left\|u_{0}\right\|_{Z}$ and $\left\|\left\{L\left(u_{0}\right) M^{-1}\right\}^{\gamma} u_{0}\right\|_{X}$.

\section{QUASILINEAR ELLIPTIC-PARABOLIC EQUATIONS}

As an application of our abstract results, we shall consider the Cauchy problem of a quasilinear elliptic-parabolic equation of the form

$$
\begin{cases}\frac{\partial}{\partial t} m(x) v=\nabla \cdot\{a(x, m(x) v) \nabla v\}+f(x, m(x) v) & \text { in }(0, T] \times \Omega \\ v=0 & \text { on }(0, T] \times \partial \Omega \\ m(x) v(x, 0)=u_{0}(x) & \text { in } \Omega\end{cases}
$$


in a bounded region $\Omega \subset \mathbb{R}^{n}$ of $\mathcal{C}^{2}$ class. In this paper we handle the cases where $n=1,2$, and 3. $m(x) \geq 0$ is a nonnegative function such that

$$
m(x) \in \mathrm{C}^{1}(\bar{\Omega}) \text { when } n=1, \quad m(x) \in \mathrm{C}^{2}(\bar{\Omega}) \text { when } n=2,3 .
$$

$a(x, u)$ is a real valued smooth function defined for $(x, u) \in \bar{\Omega} \times(\mathbb{R}+i \mathbb{R})$, and it is assumed for each $0<R<\infty$ to satisfy

$$
a(x, u) \geq \delta_{R}>0
$$

for all $x \in \bar{\Omega}$ and $u$ such that $|u| \leq R$ with some positive constant $\delta_{R} . f(x, u)$ is a smooth function of $(x, u) \in \bar{\Omega} \times(\mathbb{R}+i \mathbb{R})$. For the initial function, we assume that

$$
u_{0}=m(x) v_{0} \text { with some } v_{0} \in\left\{\begin{array}{l}
H_{0}^{1}(\Omega) \text { when } n=1, \\
H^{2}(\Omega) \cap H_{0}^{1}(\Omega) \text { when } n=2,3 .
\end{array}\right.
$$

Case when $n=1$. In this case we formulate (5.1) in the space $X=H^{-1}(\Omega)$. We take as $Z$ the Sobolev space $H^{\frac{1}{2}+\varepsilon}(\Omega)$, where $\varepsilon$ is an exponent arbitrarily fixed so that $\varepsilon \in\left(0, \frac{1}{2}\right)$. Let $u_{0} \in Z$ and $\left\|u_{0}\right\|_{Z}<R<\infty$, then $K$ is taken as $K=\left\{u \in Z ;\|u\|_{Z}<R\right\}$. By the embedding theorem, $K$ is a bounded set of $\mathrm{C}(\bar{\Omega})$. For $u \in K$, the sesquilinear form

$$
a\left(u ; w_{1}, w_{2}\right)=\int_{\Omega} a(x, u(x)) \nabla w_{1}(x) \cdot \nabla \overline{w_{2}(x)} d x, \quad w_{1}, w_{2} \in H_{0}^{1}(\Omega)
$$

is defined. According to the Lax-Milgram theorem (see e. g. [13, Chap. 2,Thm. $9.1])$, this sesquilinear form determines under (5.3) a closed linear operator $L(u)$ in $H^{-1}(\Omega)$ with the domain $\mathcal{D}(L(u))=H_{0}^{1}(\Omega)=\mathcal{D}_{L}$ which is in fact an isomorphism from $\mathcal{D}_{L}$ to $X$. Implicitly, $L(u)$ is the differential operator $-\nabla \cdot\{a(x, u) \nabla w\}$. We define $M$ as the multiplication operator of the function $m(x)$, in view of (5.2), $M$ is a bounded linear operator on both $X$ and $H^{1}(\Omega)$, that is $M \in \mathcal{L}(X) \cap \mathcal{L}\left(H^{1}(\Omega)\right)$. $F(u)$ is defined by

$$
F(u)=f(x, u(x)), \quad u \in K
$$

In this way, (5.1) is written as the Cauchy problem of an abstract equation of the form (D.E) in $X$. Let us now verify all the assumptions (D.Ai,ii,iii) and (D.F) in Section 4. It is already known by [3, Example 6.3, (6.7)] that (D.A.i) is fulfilled with a suitable sectorial domain $\Sigma$ and $\kappa=0$.

In order to verify (D.A.ii), we use the interpolation property that $H^{\frac{1}{2}+\varepsilon}(\Omega)=$ $\left[L^{2}(\Omega), H^{1}(\Omega)\right]_{\frac{1}{2}+\varepsilon}$. Then,

$$
\begin{aligned}
\left\|M(\lambda M-L(u))^{-1}\right\|_{\mathcal{L}(X, Z)} & \\
& \leq C\left\|M(\lambda M-L(u))^{-1}\right\|_{\mathcal{L}\left(X, H^{1}\right)}^{\frac{1}{2}+\varepsilon}\left\|M(\lambda M-L(u))^{-1}\right\|_{\mathcal{L}\left(X, L^{2}\right)}^{\frac{1}{2}-\varepsilon} .
\end{aligned}
$$

But, from $[3,(6.6)$ and (6.8)] it is known that

$$
\begin{gathered}
\left\|(\lambda M-L(u))^{-1}\right\|_{\mathcal{L}\left(X, H^{1}\right)} \leq C, \quad \lambda \notin \Sigma, u \in K, \\
\left\|M(\lambda M-L(u))^{-1}\right\|_{\mathcal{L}\left(X, L^{2}\right)} \leq \frac{C}{(|\lambda|+1)^{\frac{1}{2}}}, \quad \lambda \notin \Sigma, u \in K,
\end{gathered}
$$


therefore we obtain that

$$
\left\|M(\lambda M-L(u))^{-1}\right\|_{\mathcal{L}(X, Z)} \leq C(|\lambda|+1)^{\frac{\varepsilon}{2}-\frac{1}{4}}, \quad \lambda \notin \Sigma, u \in K .
$$

This shows that (D.A.ii) is valid with $\rho=\frac{3}{4}+\frac{\varepsilon}{2}$.

Verification of (D.A.iii) is very easy. Indeed, we have

$$
\begin{array}{r}
\left\langle L(u)\left\{L(u)^{-1}-L(\widetilde{u})^{-1}\right\} f, w\right\rangle_{H^{-1} \times H_{0}^{1}}=\left\langle\{L(\widetilde{u})-L(u)\} L(\widetilde{u})^{-1} f, w\right\rangle_{H^{-1} \times H_{0}^{1}} \\
=\int_{\Omega}\{a(x, \widetilde{u}(x))-a(x, u(x))\} \nabla L(\widetilde{u})^{-1} f \cdot \nabla w d x, \quad f \in X, w \in \mathcal{D}_{L} .
\end{array}
$$

Therefore, since

$$
\|a(x, \widetilde{u})-a(x, u)\|_{\mathcal{e}} \leq C\|\widetilde{u}-u\|_{\mathcal{e}} \leq C\|\widetilde{u}-u\|_{Z}, \quad \widetilde{u}, u \in K,
$$

(D.A.iii) follows immediately.

Verification of (D.F) is also very easy. Finally, (5.4) implies that $u_{0}$ belongs to $\mathcal{D}\left(L\left(u_{0}\right) M^{-1}\right)$, that is $u_{0}$ satisfies (D.In) with $\gamma=1$. (D.Ex) is then fulfilled with $\kappa=0, \rho=\frac{3}{4}+\frac{\varepsilon}{2}$, and $\gamma=1$.

We have thus shown that, under (5.2), (5.3) and (5.4), we can apply the Theorem 4.1 to the problem (5.1).

Case when $n=2,3$. In this case, we take as $X$ the space $L^{2}(\Omega)$ and as $Z$ the Sobolev space $H^{\frac{n}{2}+\varepsilon}(\Omega)$, where $\varepsilon$ is an exponent arbitrarily fixed so that $\varepsilon \in\left(0, \frac{1}{2}\right)$. Let $u_{0} \in Z$ and $\left\|u_{0}\right\|_{Z}<R<\infty$. Then $K$ is taken as $K=\left\{u \in Z ;\|u\|_{Z}<R\right\}$. $K$ is a bounded set of $\mathrm{C}(\bar{\Omega})$. For $u \in K$, the linear operator $L(u)$ is defined by $L(u) w=-\nabla \cdot\{a(x, u(x)) \nabla w\}+c w$ with $\mathcal{D}(L(u))=H^{2}(\Omega) \cap H_{0}^{1}(\Omega)$, where $c$ is some sufficiently large constant for which all the arguments below are true. $L(u)$ is a positive definite self-adjoint operator of $X$, the domain $\mathcal{D}(L(u)) \equiv \mathcal{D}_{L}$ being independent of $u$. The following estimate also holds

$$
\|w\|_{H^{2}} \leq C\|L(u) w\|_{L^{2}}, \quad w \in \mathcal{D}_{L}, u \in K .
$$

$M$ is a multiplication operator of $m(x)$; in view of (5.2), $M$ is seen to be a bounded linear operator on both $X$ and $H^{2}(\Omega)$. $F(u)$ is defined by $F(u)=c u+f(x, u), u \in$ $K$. Then (5.1) is formulated as the Cauchy problem of an abstract equation of the form (D.E) in $X$.

In the present case we have to assume in addition to (5.2) the following order condition

$$
|\nabla m(x)| \leq C m(x)^{\zeta}, \quad x \in \bar{\Omega}
$$

with some suitable exponent $\zeta \in[0,1)$ which will be specified below. As shown in [3, Example 6.3], condition (5.6) yields that

$$
\left\|M(\lambda M-L(u))^{-1}\right\|_{\mathcal{L}(X)} \leq C(|\lambda|+1)^{-\frac{1}{2-\zeta}}, \quad \lambda \notin \Sigma, u \in K
$$

with some suitable sectorial domain $\Sigma, 0<\phi<\frac{\pi}{2}$. Therefore, with $\kappa=\frac{1-\zeta}{2-\zeta}$,

$$
\begin{gathered}
\left\|M(\lambda M-L(U))^{-1}\right\|_{\mathcal{L}(X)} \leq C(|\lambda|+1)^{\kappa-1}, \quad \lambda \notin \Sigma, u \in K, \\
\left\|(\lambda M-L(u))^{-1}\right\|_{\mathcal{L}\left(X, H^{2}\right)} \leq C(|\lambda|+1)^{\kappa}, \quad \lambda \notin \Sigma, u \in K .
\end{gathered}
$$


By the interpolation property that $Z=H^{\frac{n}{2}+\varepsilon}(\Omega)=\left[L^{2}(\Omega), H^{2}(\Omega)\right]_{\frac{n}{4}+\frac{\varepsilon}{2}}$, it follows that

$$
\begin{aligned}
\| M(\lambda M-L(u))^{-1} & \|_{\mathcal{L}(X, Z)} \\
& \leq C\left\|M(\lambda M-L(u))^{-1}\right\|_{\mathcal{L}(X, X)}^{1-\frac{n}{4}-\frac{\varepsilon}{2}}\left\|M(\lambda M-L(u))^{-1}\right\|_{\mathcal{L}\left(X, H^{2}\right)}^{\frac{n}{4}+\frac{\varepsilon}{2}}
\end{aligned}
$$

(5.7) and (5.8) yield that

$$
\left\|M(\lambda M-L(u))^{-1}\right\|_{\mathcal{L}(X, Z)} \leq C(|\lambda|+1)^{-1+\frac{n}{4}+\frac{\varepsilon}{2}+\kappa}, \quad \lambda \in \Sigma, u \in K .
$$

Therefore, (D.A.ii) is fulfilled with $\rho=\frac{n}{4}+\frac{\varepsilon}{2}+\kappa$.

(D.A.iii) is verified directly from

$$
\begin{aligned}
& L(u)\left\{L(u)^{-1}-L(\widetilde{u})^{-1}\right\} f=\{L(\widetilde{u})-L(u)\} L(\widetilde{u})^{-1} f \\
& \quad=\{a(x, u)-a(x, \widetilde{u})\} \Delta L(\widetilde{u})^{-1} f+\nabla\{a(x, u)-a(x, \widetilde{u})\} \cdot \nabla L(\widetilde{u})^{-1} f .
\end{aligned}
$$

Note that the following estimate

$$
\|a(x, u)-a(x, \widetilde{u})\|_{Z} \leq C\|u-\widetilde{u}\|_{Z}, \quad u, \widetilde{u} \in K
$$

is verified by using the theory of Sobolev spaces (cf. [7]).

(D.F) is also verified immediately. (5.4) implies that $u_{0} \in \mathcal{D}\left(L\left(u_{0}\right) M^{-1}\right)$, that is (D.In) is valid with $\gamma=1$. Therefore, by simple calculations, (D.Ex) is shown to be valid, provided that

$$
\frac{16+2 n+4 \varepsilon}{20+n+2 \varepsilon}<\zeta<1, \quad n=2,3 .
$$

Thus, under (5.2), (5.3), (5.4), (5.6), and (5.9), Theorem 4.1 is applicable to the problem (5.1).

Remark 5.1. According to Favini et al. [4], (D.A.i) is valid even in the space $L^{p}(\Omega), 1<p<\infty$. If we utilize these results, it is equally possible to handle the problem (5.1) in $L^{p}$ spaces.

\section{Quasilinear Elliptic-Parabolic Systems}

In this section let us consider an elliptic-parabolic system of the form

$$
\left\{\begin{array}{rrr}
\frac{\partial u}{\partial t}=\frac{\partial}{\partial x}\left\{a(x, u) \frac{\partial}{\partial x} u+b(x, u) \frac{\partial}{\partial x} v\right\}+f(x, u) & \text { in }(0, T] \times \Omega \\
0=\frac{\partial}{\partial x}\left\{c(x, u) \frac{\partial}{\partial x} u+d(x, u) \frac{\partial}{\partial x} v\right\}+g(x, u) & \text { in }(0, T] \times \Omega \\
u=v=0 & \text { on }(0, T] \times \partial \Omega \\
u(x, 0)=u_{0}(x) & \text { in } \Omega
\end{array}\right.
$$

in a bounded open interval $\Omega=(0, \ell)$.

$a(x, u), b(x, u), c(x, u)$, and $d(x, u)$ are all real valued smooth functions of variables $(x, u) \in \bar{\Omega} \times(\mathbb{R}+i \mathbb{R})$. It is assumed that, for each $0<R<\infty$, there exists 
some positive constant $\delta_{R}>0$ dependent on $R$ such that the following estimate holds:

(6.2) $a(x, u) \xi^{2}+(b(x, u)+c(x, u)) \xi \eta+d(x, u) \eta^{2} \geq \delta_{R}\left(\xi^{2}+\eta^{2}\right), \quad \forall \xi, \forall \eta \in \mathbb{R}$ for all $x \in \bar{\Omega}$ and $u$ such that $|u| \leq R$.

$f(x, u)$ and $g(x, u)$ are given smooth functions of variable $(x, u) \in \bar{\Omega} \times(\mathbb{R}+i \mathbb{R})$. Initial value $u_{0}$ is assumed to satisfy

$$
u_{0} \in H_{0}^{1}(\Omega) .
$$

We intend to formulate the problem (6.1) in a product space

$$
X=\left\{\left(\begin{array}{l}
f \\
g
\end{array}\right) ; f, g \in H^{-1}(\Omega)\right\} .
$$

As $Z$ we take the space

$$
Z=\left\{\left(\begin{array}{l}
u \\
0
\end{array}\right) ; u \in H^{\frac{1}{2}+\varepsilon}(\Omega)\right\},
$$

where $\varepsilon$ is an arbitrarily fixed exponent so that $\varepsilon \in\left(0, \frac{1}{2}\right)$, in view of the fact that $H^{\frac{1}{2}+\varepsilon}(\Omega) \subset \mathcal{e}(\bar{\Omega})$. Then $K$ is taken as

$$
K=\left\{\left(\begin{array}{l}
u \\
0
\end{array}\right) \in Z ;\|u\|_{H^{\frac{1}{2}+\varepsilon}}<R\right\}
$$

with some fixed $0<R<\infty$ such that $\left\|u_{0}\right\|_{H^{\frac{1}{2}+\varepsilon}}<R, K$ being a bounded subset of $\mathrm{C}(\bar{\Omega})$. For $U=\left(\begin{array}{l}u \\ 0\end{array}\right) \in K$, a linear operator $L(U)$ acting in $X$ is defined by

$$
L(U) V=-\left(\begin{array}{cc}
D_{x}\left\{a(x, u) D_{x} \cdot\right\} & D_{x}\left\{b(x, u) D_{x} \cdot\right\} \\
D_{x}\left\{c(x, u) D_{x} \cdot\right\} & D_{x}\left\{d(x, u) D_{x} \cdot\right\}
\end{array}\right)\left(\begin{array}{c}
\widetilde{u} \\
\widetilde{v}
\end{array}\right), \quad V=\left(\begin{array}{c}
\widetilde{u} \\
\widetilde{v}
\end{array}\right)
$$

where $D_{x}=\frac{\partial}{\partial x}$, with the domain

$$
\mathcal{D}(L(U)) \equiv \mathcal{D}_{L}=\left\{\left(\begin{array}{l}
\widetilde{u} \\
\widetilde{v}
\end{array}\right) ; \widetilde{u}, \widetilde{v} \in H_{0}^{1}(\Omega)\right\} .
$$

$F(U): K \rightarrow X$ is defined by

$$
F(U)=\left(\begin{array}{l}
f(x, u) \\
g(x, u)
\end{array}\right), \quad U=\left(\begin{array}{l}
u \\
0
\end{array}\right) \in K .
$$

Finally, $M$ is defined as the projection on $X$ such that

$$
M\left(\begin{array}{l}
f \\
g
\end{array}\right)=\left(\begin{array}{l}
f \\
0
\end{array}\right), \quad\left(\begin{array}{l}
f \\
g
\end{array}\right) \in X .
$$

Obviously, $M$ maps $\mathcal{D}_{L}$ into $Z$.

In this way we are led to the following abstract formulation of (6.1)

$$
\left\{\begin{array}{l}
\frac{d M V}{d t}+L(M V) V=F(M V), \quad 0<t \leq T, \\
M V(0)=U_{0}=\left(\begin{array}{c}
u_{0} \\
0
\end{array}\right)
\end{array}\right.
$$


in the space $X$. Let us show that the Theorem 4.1 is applicable to this Cauchy problem.

In order to verify (D.A.i) we have to consider sesquilinear forms on $\mathcal{D}_{L}$ :

$$
\begin{aligned}
A_{\lambda}\left(U ; V_{1}, V_{2}\right)= & \int_{\Omega}\left\{a(x, u) D_{x} \widetilde{u}_{1} \cdot D_{x} \overline{\widetilde{u}_{2}}+b(x, u) D_{x} \widetilde{v}_{1} \cdot D_{x} \overline{\widetilde{u}_{2}}\right. \\
& \left.+c(x, u) D_{x} \widetilde{u}_{1} \cdot D_{x} \widetilde{\widetilde{v}_{2}}+d(x, u) D_{x} \widetilde{v}_{1} \cdot D_{x} \overline{\widetilde{v}_{2}}\right\} d x \\
& \quad-\lambda \int_{\Omega} \widetilde{u}_{1} \overline{\widetilde{u}_{2}} d x, \quad V_{1}=\left(\begin{array}{c}
\widetilde{u}_{1} \\
\widetilde{v}_{1}
\end{array}\right), \quad V_{2}=\left(\begin{array}{c}
\widetilde{u}_{2} \\
\widetilde{v}_{2}
\end{array}\right) \in \mathcal{D}_{L},
\end{aligned}
$$

where $U=\left(\begin{array}{l}u \\ 0\end{array}\right) \in K$ and $\lambda \in \mathbb{C}$. It is immediate to see that

$$
\left|A_{\lambda}\left(U ; V_{1}, V_{2}\right)\right| \leq C(|\lambda|+1)\left\|V_{1}\right\|_{\mathcal{D}_{L}}\left\|V_{2}\right\|_{\mathcal{D}_{L}}, \quad V_{1}, V_{2} \in \mathcal{D}_{L}
$$

In addition, we verify that

$$
\begin{aligned}
\Re \mathrm{e} A_{\lambda}(U ; V, V) & =-\Re \mathrm{e} \lambda \int_{\Omega}|\widetilde{u}|^{2} d x+\int_{\Omega}\left\{a(x, u)\left|D_{x} \widetilde{u}\right|^{2}+d(x, u)\left|D_{x} \widetilde{v}\right|^{2}\right. \\
& \left.+(b(x, u)+c(x, u))\left(\Re \mathrm{e} D_{x} \widetilde{u} \cdot \Re \mathrm{e} D_{x} \widetilde{v}+\Im \mathrm{m} D_{x} \widetilde{u} \cdot \Im \mathrm{m} D_{x} \widetilde{v}\right)\right\} d x
\end{aligned}
$$

and

$$
\begin{aligned}
\Im \mathrm{m} A_{\lambda}(U, V, V) & =-\Im \mathrm{m} \lambda \int_{\Omega}|\widetilde{u}|^{2} d x \\
+ & \int_{\Omega}(b(x, u)-c(x, u))\left(\Re \mathrm{e} D_{x} \widetilde{u} \cdot \Im \mathrm{m} D_{x} \widetilde{v}-\Im \mathrm{m} D_{x} \widetilde{u} \cdot \Re \mathrm{e} D_{x} \widetilde{v}\right) d x .
\end{aligned}
$$

Then, by (6.2) there exists $\delta>0$ such that

$$
\begin{gathered}
\Re \mathrm{e} A_{\lambda}(U ; V, V) \geq \int_{\Omega}\left\{\delta\left(\left|D_{x} \widetilde{u}\right|^{2}+\left|D_{x} \widetilde{v}\right|^{2}\right)-\Re \operatorname{e} \lambda|\widetilde{u}|^{2}\right\} d x, \quad V \in \mathcal{D}_{L}, \\
\left|\Im \mathrm{m} A_{\lambda}(U ; V, V)\right| \geq \int_{\Omega}\left\{|\Im \mathrm{m} \lambda||\widetilde{u}|^{2}-C_{R}\left(\left|D_{x} \widetilde{u}\right|^{2}+\left|D_{x} \widetilde{v}\right|^{2}\right)\right\} d x, \quad V \in \mathcal{D}_{L},
\end{gathered}
$$

here $C_{R}$ denotes a constant

$$
C_{R}=\sup _{x \in \bar{\Omega}, U \in K}|b(x, u(x))-c(x, u(x))| .
$$

Let us introduce a parameter $0<\theta<1$, and observe that

$$
\begin{aligned}
& \left|A_{\lambda}(U ; V, V)\right| \geq(1-\theta) \Re \mathrm{e} A_{\lambda}(U ; V, V)+\theta\left|\Im \mathrm{m} A_{\lambda}(U ; V, V)\right| \\
& \quad \geq\left((1-\theta) \delta-\theta C_{R}\right)\left(\left\|D_{x} \widetilde{u}\right\|_{L^{2}}^{2}+\left\|D_{x} \widetilde{v}\right\|_{L^{2}}^{2}+(\theta|\Im \mathrm{m} \lambda|-(1-\theta) \Re \mathrm{e} \lambda)\|\widetilde{u}\|_{L^{2}}^{2} .\right.
\end{aligned}
$$

Then, if $\theta>0$ is sufficiently small so that $(1-\theta) \delta-\theta C_{R} \geq \frac{\delta}{2}$, and if $\lambda$ is taken in such a way that

then

$$
\lambda \notin \Sigma=\{\lambda \in \mathbb{C} ;|\arg \lambda|<\phi\}, \operatorname{Tan}^{-1} \frac{1-\theta}{\theta}<\phi<\frac{\pi}{2},
$$

$$
\left|A_{\lambda}(U ; V, V)\right| \geq \delta^{\prime}\left(\|V\|_{\mathcal{D}_{L}}^{2}+|\lambda|\|M V\|_{L^{2}}^{2}\right), \quad V \in \mathcal{D}_{L}, U \in K
$$


with some positive constant $\delta^{\prime}>0$. Here we used the Poincaré inequality

$$
\|\widetilde{u}\|_{L^{2}} \leq C\left\|D_{x} \widetilde{u}\right\|_{L^{2}}, \quad \widetilde{u} \in H_{0}^{1}(\Omega)
$$

In view of (6.6) and (6.7), we can now apply the Lax-Milgram theorem (see e. g. [13, Chap. 2,Thm. 9.1,Rem. 9.3]). Let $\lambda \notin \Sigma$, then for a given $F \in X$, the problem

$$
A_{\lambda}(U ; V, W)=\langle F, W\rangle_{X \times \mathcal{D}_{L}}, \quad \forall W \in \mathcal{D}_{L}
$$

has a unique solution $V \in \mathcal{D}_{L}$ with the estimates

$$
\begin{gathered}
\|V\|_{\mathcal{D}_{L}} \leq\left(1 / \delta^{\prime}\right)\|F\|_{X}, \\
|\lambda|^{\frac{1}{2}}\|M V\|_{L^{2}} \leq\left(1 / \delta^{\prime}\right)\|F\|_{X} .
\end{gathered}
$$

Since

$$
A_{\lambda}(U ; V, W)=-\langle(\lambda M-L(U)) V, W\rangle_{X \times \mathcal{D}_{L}}, \quad \forall W \in \mathcal{D}_{L},
$$

it follows that for a given $F \in X$, the problem

$$
-(\lambda M-L(U)) V=F
$$

has a unique solution with the estimates (6.8) and (6.9). This then means that $\lambda \in \rho_{M}(L(U))$ and the resolvent satisfies the estimates

$$
\begin{gathered}
\left\|(\lambda M-L(U))^{-1} F\right\|_{\mathcal{D}_{L}} \leq\left(1 / \delta^{\prime}\right)\|F\|_{X}, \quad F \in X, \\
|\lambda|^{\frac{1}{2}}\left\|(\lambda M-L(U))^{-1} F\right\|_{L^{2}} \leq\left(1 / \delta^{\prime}\right)\|F\|_{X}, \quad F \in X .
\end{gathered}
$$

In this way we have verified that

$$
\sigma_{M}(L(U)) \subset \Sigma, \quad U \in K
$$

Therefore it suffices to verify the estimate (D.A.i). But by (6.10) it is now seen that

$$
\begin{aligned}
& \left\|\lambda M(\lambda M-L(U))^{-1} F\right\|_{X}=\left\|F+L(U)(\lambda M-L(U))^{-1} F\right\|_{X} \\
& \quad \leq\|F\|_{X}+C\left\|(\lambda M-L(U))^{-1} F\right\|_{\mathcal{D}_{L}} \leq C\left(1+\left(1 / \delta^{\prime}\right)\right)\|F\|_{X}, \quad F \in X .
\end{aligned}
$$

Hence, (D.A.i) is fulfilled with the domain $\Sigma$ determined above and $\kappa=0$.

To verify (D.A.ii) we use the interpolation property that $H^{\frac{1}{2}+\varepsilon}(\Omega)=\left[L^{2}(\Omega)\right.$, $\left.H^{1}(\Omega)\right]_{\frac{1}{2}+\varepsilon}$. Then, by the same argument as for $(5.5),(6.10)$ and $(6.11)$ yield that

$$
\left\|(\lambda M-L(U))^{-1}\right\|_{\mathcal{L}(X, Z)} \leq C(|\lambda|+1)^{\frac{\varepsilon}{2}-\frac{1}{4}}, \quad \lambda \notin \Sigma .
$$

Hence, (D.A.ii) is valid with $\rho=\frac{3}{4}+\frac{\varepsilon}{2}$.

(D.A.iii) is verified directly as in the previous section by using the expression

$$
\begin{gathered}
L\left(U_{1}\right)\left\{L\left(U_{1}\right)^{-1}-L\left(U_{2}\right)^{-1}\right\}=\left\{L\left(U_{2}\right)-L\left(U_{1}\right)\right\} L\left(U_{2}\right)^{-1} \\
=\left(\begin{array}{cc}
D_{x}\left\{\left[a\left(x, u_{1}\right)-a\left(x, u_{2}\right)\right] D_{x} \cdot\right\} & D_{x}\left\{\left[b\left(x, u_{1}\right)-b\left(x, u_{2}\right)\right] D_{x} \cdot\right\} \\
D_{x}\left\{\left[c\left(x, u_{1}\right)-c\left(x, u_{2}\right)\right] D_{x} \cdot\right\} & D_{x}\left\{\left[d\left(x, u_{1}\right)-d\left(x, u_{2}\right)\right] D_{x} \cdot\right\}
\end{array}\right) L\left(U_{2}\right)^{-1}, \\
U_{i}=\left(\begin{array}{c}
u_{i} \\
0
\end{array}\right) \in K(i=1,2) .
\end{gathered}
$$


(D.F) is also verified directly. Since $\mathcal{D}\left(L\left(U_{0}\right) M^{-1}\right)=M\left(\mathcal{D}_{L}\right)$, (6.3) together with (6.4) implies that the initial value $U_{0}$ in (6.5) belongs to $D\left(L\left(U_{0}\right) M^{-1}\right)$. Hence, (D.In) is valid with $\gamma=1$. (D.Ex) is also fulfilled as well.

In this way we conclude that, under (6.2) and (6.3), Theorem 4.1 is applied to (6.5) to obtain the existence and uniqueness of local solution.

\section{REFERENCES}

[1] C. Bardos and H. Brezis, Sur une classe de problèmes d'évolution nonlinéaires, J. Diff. Eqs. 6, 1969, 345-394.

[2] H. Brezis, On some degenerate nonlinear parabolic equations, Nonlinear Functional Analysis, Proc. Symp. Pure Math. 18, 1970, 28-38.

[3] A. Favini and A. Yagi, Multivalued linear operators and degenerate evolution equations, Ann. Mat. Pura. Appl. (IV) 163, 1993, 353-384.

[4] A.Favini, A. Lorenzi, H. Tanabe and A. Yagi, An $L^{p}$-approach to singular linear parabolic equations in bounded domains, Preprint.

[5] A. Kuttler, A degenerate nonlinear Cauchy problem, Applicable Analysis 13, 1982, 307-322.

[6] A. Kuttler, Implicit evolution equations, Appl. An. 16, 1983, 91-99.

[7] K. Osaki, T. Tsujikawa, A. Yagi and M. Mimura, Exponential attractor for a chemotaxisgrowth system of equations, Nonlinear Analysis 51, 2002, 119-144.

[8] P. E. Sobolevskii, Equations of parabolic type in Banach space, Amer. Math. Soc. Trans. Ser. $249,1966,1-62$.

[9] A. Yagi, Abstract quasilinear evolution equations of parabolic type in Banach spaces, Boll. Un. Mat. Ital. 5-B, 1991, 341-368.

[10] R. W. Carroll and R. E. Showalter, Singular and Degenerate Cauchy Problems, 1976, Academic Press, London, New York.

[11] A. Favini and A. Yagi, Degenerate Differential Equations in Banach Spaces, 1999, Marcel Dekker, New York.

[12] A. Friedman, Partial Differential Equations , 1969, Holt, Rinehart and Winston, New York.

[13] J. L. Lions et E. Magenes, Problèmes aux limites non homogènes et Applications, vol. 1 , 1968, Dunod, Paris.

[14] Yu. N. Rabotnov, Polzuchest' Elementov Konstruktsii (Creep of constructional elements), 1967, Nauka, Moscow.

[15] W. Shockley, Electrons and Holes in Semiconductors, 1950, D. Van Nostrand, Princeton, New Jersey.

[16] R. E. Showalter, Monotone Operators in Banach Space and Nonlinear Partial Differential Equations, Math. Surveys \& Monographs 49, 1997, AMS, Providence.

[17] H. Tanabe, Functional Analytic Methods for Partial Differential Equations, 1997, Marcel Dekker, New York.

(*) Department of Mathematics, University of Bologna, Piazza di Porta S. DoNATO 5, 40126 Bologna, Italia

(**) Department of Applied Physics, Osaka University, Suita, Osaka 565-0871, Japan 